\title{
A Langevin equation for high energy evolution with pomeron loops
}

\author{
E. Iancu ${ }^{1}$ and D. N. Triantafyllopoulos \\ Service de Physique Théorique, CEA/DSM/SPhT, Unité de recherche associée au \\ CNRS (URA D2306), CE Saclay, F-91191 Gif-sur-Yvette, France
}

\begin{abstract}
We show that the Balitsky-JIMWLK equations proposed to describe non-linear evolution in QCD at high energy fail to include the effects of fluctuations in the gluon number, and thus to correctly describe both the low density regime and the approach towards saturation. On the other hand, these fluctuations are correctly encoded (in the limit where the number of colors is large) in Mueller's color dipole picture, which however neglects saturation. By combining the dipole picture at low density with the JIMWLK evolution at high density, we construct a generalization of the Balitsky hierarchy which includes the particle number fluctuations, and thus the pomeron loops. After an additional coarse-graining in impact parameter space, this hierarchy is shown to reduce to a Langevin equation in the universality class of the stochastic Fisher-Kolmogorov-Petrovsky-Piscounov (sFKPP) equation. This equation implies that the non-linear effects in the evolution become important already in the high momentum regime where the average density is small, which signals the breakdown of the BFKL approximation.
\end{abstract}

$\overline{1}$ Membre du Centre National de la Recherche Scientifique (CNRS), France. 


\section{Contents}

1 Introduction 2

$\begin{array}{lll}2 & \text { Phvsical motivation } & 8\end{array}$

3 The Balitskv-JIMWLK equations and bevond 11

$4 \quad$ A tov model borrowed from statistical physics 20

$5 \quad$ Fluctuations and evolution in the dipole picture 25

$6 \quad$ Fluctuations and saturation in dipole-CGC scattering 30

6.1 From dipole densities to scattering amplitudes 31

6.2 The equations for the scattering amplitudes 33

\begin{tabular}{|ll}
6.3 & The Langevin equation \\
\hline
\end{tabular}

\begin{tabular}{lll}
\hline$\quad$ Phvsical discussion & 40
\end{tabular}

\begin{tabular}{|ll}
\hline 7.1 & Relation with the sFKPP equation
\end{tabular}

7.2 $\quad$ Some results from sFKPP equation and their consequences for QCD 43

7.3 Front diffusion and the breakdown of the BFKL approximation 46

A A one-dimensional reaction-diffusion model 50

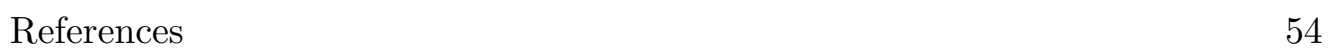

\section{Introduction}

Over the last decade, much progress has been realized towards understanding the dynamics of QCD at high energies, in the vicinity of the unitarity limit. New theoretical approaches have been developed which encompass and extend the linear BFKL equation [1] originally proposed to describe high energy evolution in the leading-logarithmic approximation (with respect to $\ln s$ ), as well as the earlier attempts to improve over the BFKL equation by including unitarity corrections (or gluon saturation) in the form of non-linear terms $[2,3,4]$.

In the mid nineties, Al Mueller [5,6] has given an elegant construction of the BFKL wavefunction of an energetic hadron, the 'color dipole picture', which exploits the large $-N_{c}$ approximation (with $N_{c}$ the number of colors) to 
replace gluons by color dipoles as the effective degrees of freedom at small $x$, and gluon radiation by dipole splitting as the mechanism for evolution. The average number of dipoles obeys BFKL equation, but the overall description goes beyond the original BFKL approach by including the many-body correlations generated through dipole splitting. Accordingly, the evolution is expressed as a hierarchy of equations for the dipole density correlations. The dipole picture cannot describe interactions among the dipoles in the wavefunction (since such interactions would lead to color configurations with higher multipolar moments), so it cannot accommodate gluon saturation. But it can be used to study the onset of unitarity corrections in the elastic scattering between two sets of dipoles which have evolved separately, provided the collision is viewed in the center-of-mass frame and the total energy is not too large [6]. (The unitarization is brought in by the multiple scattering of several pairs of dipoles from the two colliding systems.) More generally, the dipole picture can be used to describe the dilute (in the sense of non-saturated) part of a hadron wavefunction up to arbitrarily high energies, and this is how we shall actually use it throughout this paper.

A different formalism to study unitarization at high energy, in which the unitarity corrections are encoded directly in the evolution equations, has been developed by Balitsky [7]. This involves a hierarchy of equations describing the evolution of the scattering amplitudes for the collision between a generic target and a set of relatively simple 'projectiles', which transform into each other under the evolution. The target is not evolving, but is generally taken to be a high-density system, or 'color glass condensate', represented by a strong color field. The evolution is achieved by boosting the projectile, but in such a way that the latter remains non-saturated, which implicitly restricts the maximal energy allowed. Thus, the evolution of the projectile wavefunction is in fact linear - for a dipole projectile and in the large $-N_{c}$ limit, this is precisely the dipole picture discussed above ${ }^{2}-$, but Balitsky equations are nevertheless non-linear, since written for the scattering amplitudes : the nonlinear effects correspond to multiple scattering between the components of the projectile and the color field in the target.

Alternatively, one can keep the projectile unevolved, but use the increase in the energy in order to boost the target, and then study the small- $x$ evolution of the strong color fields there. In doing so, some of the non-linear effects which were interpreted as multiple scattering from the perspective of projectile evolution will now appear as saturation effects in the evolution of the target. But to study this, one needs a formalism capable to deal with non-linear effects in the evolution of the wavefunction. For the reasons explained before,

$\overline{2}$ Recently, it has been explicitly shown, by Levin and Lublinsky [8], that the large$N_{c}$ version of the Balitsky equations follows directly from the dipole picture for the projectile, after allowing for multiple interactions with the target. 
this cannot be a large $-N_{c}$ formalism in terms of dipoles, but rather it must allow for all the possible color multipoles, and the simplest and most natural way to do so is to work in terms of gluons. So far, the only formalism of this type is the color glass condensate (CGC) $[9,10,11,12,13,14]$ (for reviews see Ref. [15]), in which the small- $x$ gluons are described as classical color fields generated by color sources at larger values of $x$, which are randomly distributed (in color and coordinate space) with a weight function which evolves with the energy. This evolution is governed by a functional Fokker-Planck equation for the weight function, the JIMWLK equation $[12,13,16]$, which is equivalent to an infinite hierarchy of equations for the correlation functions of the classical color fields. When applied to the scattering between a simple projectile and the CGC, the JIMWLK evolution reproduces Balitsky equations for the scattering amplitudes. In what follows, we shall refer to these equations as the BalitskyJIMWLK equations.

The Balitsky-JIMWLK equations will be further discussed in Sect. 3 below, but here we would like to emphasize a few important points: i) The evolution described by these equations is stochastic: Through the non-linear terms present in these equations, $n$-point functions with different values of $n$ will mix under the evolution, thus generating new correlations with increasing energy. ii) The non-linear terms are associated with the presence of strong classical fields (the field of the target in the approach by Balitsky, or the field created by color sources at higher values of $x$ in the CGC). Therefore, in the dilute regime where the fields are weak, the equations can be linearized, and once we do so, they decouple from each other (so that the various $n$-point functions obey independently the BFKL evolution). This should be contrasted to the dipole picture, where correlations beyond the BFKL equation appear already in the dilute regime. iii) Even in the full equations with the non-linear terms included, the stochastic aspects turn out to become inessential at large ${ }^{3}$ $N_{c}$. For instance, in the absence of correlations in the initial conditions, the large $-N_{c}$ version of the Balitsky hierarchy boils down to a single, non-linear, equation, which is deterministic. This is the equation originally derived by Kovchegov [17] and generally referred to as the Balitksy-Kovchegov (BK) equation. This suggests that the stochastic aspects of the Balitsky-JIMWLK equations are to be attributed to color fluctuations in the high-density regime, which are suppressed at large $N_{c}$, unlike the colorless fluctuations in the particle number, as encoded in the dipole picture, which are present already in the dilute regime and for large $N_{c}$.

$\overline{3}$ By "large $-N_{c}$ " we understand here the high-energy version of the large $-N_{c}$ approximation, due to Mueller [5,6], in which gluon exchanges which are suppressed by factors of $1 / N_{c}^{2}$ are still counted as leading order effects provided they are enhanced by appropriate powers of the energy. The neglected terms are those which, for a given power of the energy, are suppressed by higher powers of $1 / N_{c}^{2}$ than the leading-order terms. 
The previous discussion - which will be further substantiated and illustrated with a few Feynman diagrams in Sect. 3 - points out towards an insufficiency of the Balitsky-JIMWLK equations, which fail to include the correlations associated with fluctuations in the particle number. Because of that, the mean field approximation (the BK equation) should work very well for these equations at large $N_{c}$ and for uncorrelated initial conditions (like the scattering off a large nucleus), and this is indeed what is seen in numerical studies of JIMWLK evolution [18]. So far, most studies of saturation and unitarity have in fact focused on the BK equation, which is much easier to handle in practice than the general equations, and whose properties are by now rather well understood $[19,20,21,22,23,24,25,26,27,28,29]$.

However, there were indications already in the dipole picture (see especially the numerical studies by Salam $[30,31])$ that the effects of fluctuations should be important, in particular, in the evolution from a low density regime to a high density one, and thus in the approach towards saturation. Indeed, in the dilute regime, the particle number fluctuations are a natural mechanism to generate higher-point density correlations, which then evolve into the non-linear terms responsible for saturation. E.g., if one starts with an isolated gluon (or dipole) at high transverse momentum $\left(k_{\perp}\right)$, as radiated from the bulk of particles at lower $k_{\perp}$, then higher-point correlations at high $k_{\perp}$ - which were originally absent - will get built in the early stages of the evolution, as correlations in the splitting of the original dipole or its close descendence. Later on, these correlations get amplified by the standard BFKL evolution, and eventually influence the evolution of the lower-point correlations when the density is high enough.

The importance of fluctuations in the evolution towards saturation has been reiterated in recent publications [32,33,34]. In Ref. [32] it has been argued that the fluctuations slow down the approach towards the unitarity limit as compared to the mean field approximation (MFA) [14,19]. In Ref. [33], a modified MFA has been proposed, in which the unitarity constraints associated with fluctuations have been simulated by imposing a barrier at high momenta on the BFKL equation. The solution to the ensuing equation shows that fluctuations reduce considerably the rate of growth of the saturation momentum with the energy (or 'saturation exponent'), and that the corresponding meanfield limit is reached only very slowly, logarithmically, as $\alpha_{s} \rightarrow 0$. Finally, in Ref. [34], the event-by-event picture of the evolution has been considered, in which fluctuations appear naturally because, in a given event, the particle occupation numbers are discrete. Based on this picture, a correspondence has been established between high-energy evolution in QCD and a class of stochastic particle models which are actively studied in statistical physics. By using known results for the latter, the authors of Ref. [34] have confirmed the result in Ref. [33] about the slow convergence of the saturation exponent to its mean-field value, and further argued that fluctuations should eventually wash 
out the geometric scaling [35] property of the average amplitude, which was known to hold for the BK equation $[26,27,28,29]$. However, the arguments in Ref. [34] cannot predict how fast (i.e., after what energy evolution) is geometric scaling violated if one starts with an initial condition that shows scaling, nor they allow one to study the preasymptotic evolution at intermediate energies and for realistic values of $\alpha_{s}$.

More importantly, there was some confusion in Ref. [34] about what should be the right formalism to study this evolution. Namely, it has been assumed there that the correct evolution law is the one provided by the BalitskyJIMWLK equations, but this cannot be right, since these equations miss the relevant fluctuations, as we have argued above. It is then natural to ask, what is/are the equation(s) that one should solve (e.g., numerically) in order to study the interplay between fluctuations and saturation, and thus be able to verify the results in Refs. [33,34] and improve over them. In other terms, what is the correct formalism to describe evolution in QCD at high energy?

It is the main purpose of this paper to provide an answer to this question, in the form of a new set of evolution equations which generalize the (large$N_{c}$ version of the) Balitsky-JIMWLK equations by approximately including the effects of particle number fluctuations. Our basic observation is that, in fact, we do have the necessary ingredients to describe both fluctuations and saturation - these are the color dipole picture, and the JIMWLK evolution, respectively - and that, although these ingredients may look too different from each other to be simply married in a unified theoretical description of the lightcone wavefunction, they can still be naturally merged with each other in the evolution equations for scattering amplitudes. Since relying on the dipole picture, our subsequent construction is limited to the large $-N_{c}$ limit, to which we shall restrict ourselves throughout the following analysis. It remains as an open problem at this stage whether it would be possible to develop a wavefunction formalism which incorporates all these physical ingredients (e.g., through a suitable extension of the JIMWLK formalism), and thus rederive the results for scattering amplitudes that we shall present here, together with their generalization to arbitrary $N_{c}$.

Let us briefly anticipate here the main steps in these construction, and explain the other approximations that we shall need. After briefly explaining the physical picture of particle number fluctuations in the dipole picture in Sect. 2, we shall then construct, in Sect. 5, the equations which describe the evolution of the many-body density correlations in this formalism. To that aim, we shall use the recent formulation of the dipole picture in Refs. [36,37], which is more convenient for a study of fluctuations since it follows explicitly the evolution of a given configuration of $N$ dipoles. The equations that we shall obtain in Sect. 5 have been already presented by Levin and Lublinsky in a very recent publication [8], but our respective derivation will be somewhat 
different, and also better suited for our present purposes.

In the meantime, in Sect. 4, we shall also analyze a simple stochastic particle model borrowed from statistical mechanics (a zero-dimensional version of the model in Ref. [38]), which in spite of its formal simplicity has the conceptual advantage over the dipole picture to include both fluctuations and recombination (and thus saturation). The explicit manipulations permitted by the simplicity of this model will help us developping some physical intuition, and will also serve as a guidance for the corresponding manipulations in QCD, where such a unified theoretical description of the wavefunction is still lacking. A more elaborated, one-dimensional, version of the same model (the one which is actually considered in Ref. [38]) will be then discussed in the Appendix. This will give us the opportunity to introduce the stochastic Fisher-KolmogorovPetrovsky-Piscounov (sFKPP) equation, which in this context emerges as an exact equation (mathematically equivalent to the original model), and which will also emerge as an approximation to the corresponding equations in QCD.

In Sect. 6 we construct the new equations for scattering amplitudes in QCD, which are the main results of this paper. First, in Sect. 6.1, we relate dipole correlations in the target to scattering amplitudes for projectile dipoles, by using approximations which make sense in the dilute regime. This allows us to translate, in Sect. 6.2, the equations for densities constructed in Sect. 5 into corresponding equations for the scattering amplitudes. These equations are still linear, as appropriate in the low-density regime, but they include the effects of dipole number fluctuations in the target (so they form already a non-trivial hierarchy). Then, these equations are extended to the high-density regime by adding the non-linear terms expected from the JIMWLK evolution (i.e., the same non-linear terms as in the Balitsky hierarchy). Because of the combined effect of fluctuations ('gluon splitting') and recombination ('gluon merging'), the final equations generate pomeron loops through iterations. In Sect. 6.3, we show that, after a coarse-graining in the impact parameter space, the whole hierarchy can be equivalently replaced by a specific Langevin equation, which is formally the BK equation supplemented with a multiplicative noise term.

In our last section 7, we explore some physical and mathematical consequences of the new equations. In Sect. 7.1, we show that, mediating a gradient expansion of the BFKL kernel known as the 'diffusion approximation', the Langevin equation of Sect. 6 can be cast into the form of the sFKPP equation. The latter emerges as an effective equation for a variety of problems in physics, biology, and chemistry (see Refs. [39,40] for recent reviews), and it has been extensively studied in recent years in the statistical physics literature. By using some known results about this equation, we confirm (in Sect. 7.2) previous findings in Refs. [33,34], and thus conclude that the present Langevin equation is indeed the evolution law underlying the physical discus- 
sion in Ref. [34]. Finally, in Sect. 7.3, we discuss a rather dramatic consequence of the stochastic nature of the evolution, which entails the breakdown of the BFKL approximation in the high momentum regime, where the average gluon density, or the average scattering amplitude, are small. Because of fluctuations, the saturation momentum becomes a random quantity which can take different values from one event to another, or from one impact parameter to another (for the same event). Then the evolution develops high-density 'spots' where the gluons are at saturation even for relatively high transverse momenta, well above the average saturation momentum. At sufficiently high energy, all the correlations are dominated by such dense spots, and the mean field approximation (i.e., BFKL equation at high $k_{\perp}$ ) breaks down. This may look unexpected, but in fact it can be related to a similar behavior observed by Salam in his Monte-Carlo studies of the dipole picture [30,31]. The 'dense spots' were clearly seen in those numerical simulations, but of course in that context the local density was never saturating.

\section{Physical motivation}

Given a high energy hadron with rapidity $Y$, we would like to understand fluctuations in the tail of the gluon distribution at transverse momenta $k_{\perp} \gg Q_{s}(Y)$ (with $Q_{s}(Y)$ the saturation momentum), and, in particular, the influence that such fluctuations may have - through their subsequent evolution - on the approach towards saturation (or towards unitarity in the collision with an external projectile). The high- $k_{\perp}$ tail is a priori a dilute regime in which the gluon occupation numbers are small, of $\mathcal{O}(1)$, so we expect important fluctuations associated with the discreteness of the particle (here, gluon) number. Given that the gluon number density is represented, in a field theoretical formulation, by the two-point correlation function of the color fields in the hadron wavefunction, we deduce that the relevant fluctuations should be encoded in the four-point, and higher, correlations. Alternatively, and simpler, in the large $-N_{c}$ limit to which we shall stick throughout this paper, the tail of the distribution can be described in Mueller's dipole picture $[5,6]$, in which gluons are effectively replaced by quark-antiquark pairs of zero transverse size and in a color octet state. The 'color dipoles' then emerge as color singlet states built with the quark component of some gluon and the antiquark component of some other gluon with roughly the same rapidity. In this picture, which applies so long as the gluon density is low enough for saturation effects to be negligible, the (unintegrated) gluon distribution is measured by the average dipole number density $\langle n(r, Y)\rangle$ which obeys BFKL equation [1]. But the dipole picture goes beyond the strict BFKL equation by including correlations in the distribution of dipoles, namely those correlations which follow from dipole splitting in the course of the evolution. The simplest such cor- 
relations, which also encompass the particle number fluctuations that we are mainly interested in, are those encoded in the pairwise dipole number density $\left\langle n^{(2)}\left(r_{1}, r_{2}, Y\right)\right\rangle$ which is a measure of the probability to find simultaneously two dipoles, with transverse sizes $r_{1}$ and $r_{2}$, respectively (see Sect. 5 below for a more precise definition).

Since it is preferable to consider quantities which are measurable (at least, in principle), we shall study the effect of fluctuations on the scattering between the dilute hadronic target (described within the dipole picture) and an external projectile, which is itself chosen as a set of dipoles in some fixed configuration. This choice is convenient since, as well known, the dipole-dipole scattering is quasi-local in phase-space: a dipole projectile essentially counts the numbers of dipoles in the target having the same transverse size ${ }^{4}$ and impact parameter as itself. Specifically, the scattering amplitude for a single dipole can be estimated as $T(r, b, Y) \sim \alpha_{s}^{2} f(r, b, Y)$, where $r$ and $b$ denote respectively the size and the impact parameter of the projectile dipole, $\alpha_{s}^{2}$ measures the scattering amplitude for two dipoles with roughly the same size and nearby impact parameters, and $f(r, b, Y)$ is the (dimensionless) dipole occupation factor in the target, and is related to the corresponding number density via $f(r, b, Y) \sim r^{4} n(r, b, Y)$ (see Sect. 6).

To be sensitive to fluctuations, the projectile must involve at least two dipoles, and here we shall consider the case where it contains exactly two. Both dipoles are assumed to be small, so the individual scattering amplitudes $\left\langle T_{i}\right\rangle \sim \alpha_{s}^{2}\left\langle f_{i}\right\rangle$ (with $\left\langle T_{i}\right\rangle \equiv\left\langle T\left(r_{i}, b_{i}, Y\right)\right\rangle$ and $\left.i=1,2\right)$ are both small: $\left\langle T_{i}\right\rangle \ll 1$. The question is then, how large can be $\left\langle T^{(2)}\right\rangle \sim \alpha_{s}^{4}\left\langle f_{1} f_{2}\right\rangle$ (the scattering amplitude for the simultaneous scattering of both dipoles) ? Clearly, in the absence of correlations in the target, or if the correlations are only weak, one has $\left\langle f_{1} f_{2}\right\rangle \simeq\left\langle f_{1}\right\rangle\left\langle f_{2}\right\rangle$, and then $\left\langle T^{(2)}\right\rangle \simeq\left\langle T_{1}\right\rangle\left\langle T_{2}\right\rangle$ is much smaller than either $\left\langle T_{1}\right\rangle$ or $\left\langle T_{2}\right\rangle$. However, as we discuss now, there are kinematical situations in which one expects strong correlations among the dipoles in the target, which will drastically enhance $\left\langle T^{(2)}\right\rangle$ with respect to its estimate $\left\langle T_{1}\right\rangle\left\langle T_{2}\right\rangle$ for incoherent scattering.

The first such a situation is not really relevant for the main stream of this paper, and the only reason for mentioning it here is to distinguish it from the more interesting case to be discussed after. Namely, this is the situation in which both external dipoles scatter off the same dipole in the target. This is advantageous in the very dilute regime where $\langle f\rangle \ll 1$ (or $\langle T\rangle \ll \alpha_{s}^{2}$ ), since for such a process there is a single low-density penalty factor, and the corresponding contribution $\left\langle T^{(2)}\right\rangle \sim \alpha_{s}^{4}\langle f\rangle$ is indeed much larger than the uncorrelated

$\overline{4}$ More precisely, (quasi)locality in the dipole size holds so long as the dipole distribution in the target is in a genuine BFKL regime, i.e., it is characterized by some 'anomalous dimension'; see Sect. 6 for details. 
piece $\sim \alpha_{s}^{4}\langle f\rangle^{2}$. On the other hand, in view of the previous considerations on the quasi-locality of dipole-dipole scattering, it is clear that this situation requires a rather fine tuning between both the transverse sizes and the impact parameters of the two incoming dipoles $\left(r_{1} \sim r_{2}\right.$ and $\left.b_{1} \sim b_{2}\right)$, which as we shall see is not an interesting configuration for the evolution of the gluon distribution to high energies.

The second situation, which is more relevant for our purposes here, is the one in which the two dipoles making the projectile are contiguous in transverse space, meaning that the quark leg of one dipole is close to the antiquark leg of the other. If we use $\boldsymbol{x}_{1}$ and $\boldsymbol{y}_{1}\left(\boldsymbol{x}_{2}\right.$ and $\left.\boldsymbol{y}_{2}\right)$ to denote the transverse positions of the quark and respectively antiquark leg of the first (second) dipole, then contiguous configurations correspond to either $\boldsymbol{y}_{1} \approx \boldsymbol{x}_{2}$ or to $\boldsymbol{x}_{1} \approx \boldsymbol{y}_{2}$. Such configurations are interesting because the scattering amplitude $\left\langle T^{(2)}\right\rangle$ for two contiguous dipoles enters the evolution equation for the scattering amplitude of a single dipole in the regime where unitarity corrections become important (see Sect. 3). The reason why $\left\langle T^{(2)}\right\rangle$ is enhanced as compared to $\langle T\rangle^{2}$ for such configurations has to do with the dynamics of the evolution in the dipole picture: When increasing rapidity from $Y$ to $Y+d Y$, a dipole with legs at $\boldsymbol{x}$ and $\boldsymbol{y}$ can evolve by radiating one soft (i.e., small-x) gluon located at $\boldsymbol{z}$, a process which at large $-N_{c}$ is tantamount to the original dipole $(\boldsymbol{x}, \boldsymbol{y})$ splitting into two new dipoles $(\boldsymbol{x}, \boldsymbol{z})$ and $(\boldsymbol{z}, \boldsymbol{y})$, which are contiguous. This evolution leads to an increase in the scattering amplitude of a projectile made of two contiguous dipoles (with appropriate transverse coordinates) which is proportional to the average density $\langle n(\boldsymbol{x}, \boldsymbol{y})\rangle_{Y}$ of the parent dipole at rapidity $\mathrm{Y}$. That is, it is the dipole density $\langle n\rangle$, rather than the dipole pair density $\left\langle n^{(2)}\right\rangle$, which acts directly as a source for $\left\langle T^{(2)}\right\rangle$, and this is certainly advantageous in the dilute regime where ${ }^{5}\left\langle n^{(2)}\right\rangle \ll\langle n\rangle$.

For instance, if one starts with a single dipole $\left(\boldsymbol{x}_{0}, \boldsymbol{y}_{0}\right)$ at $Y=0$, then the only way to find a pair of dipoles after an evolution $d Y$ is that this pair be made of the dipoles $\left(\boldsymbol{x}_{0}, \boldsymbol{z}\right)$ and $\left(\boldsymbol{z}, \boldsymbol{y}_{0}\right)$, with arbitrary $\boldsymbol{z}$. More generally, the $Y-$ evolution of an arbitrary target will generate "high- $k_{\perp}$ fluctuations" (i.e., small dipoles which split off the larger, preexisting dipoles) with low occupation numbers, of $\mathcal{O}(1)$, and the only way to increase the dipole occupancy in those high $-k_{\perp}$ bins in the next few steps of the evolution is through the splitting of the original fluctuations. It is only when the average pair density $\left\langle n^{(2)}\right\rangle$ becomes large enough that the normal BFKL evolution (here, for $\left\langle n^{(2)}\right\rangle$ ) takes over, and the fluctuations (at that particular value of $k_{\perp}$ ) cease to play a role. We see that a high $-k_{\perp}$ fluctuation play the crucial role of a seed for extending the gluon distribution towards larger transverse momenta with increasing $Y$.

\footnotetext{
5 More correctly, this inequality should be written for the dimensionless occupation numbers, as $\langle f f\rangle \ll\langle f\rangle$.
} 


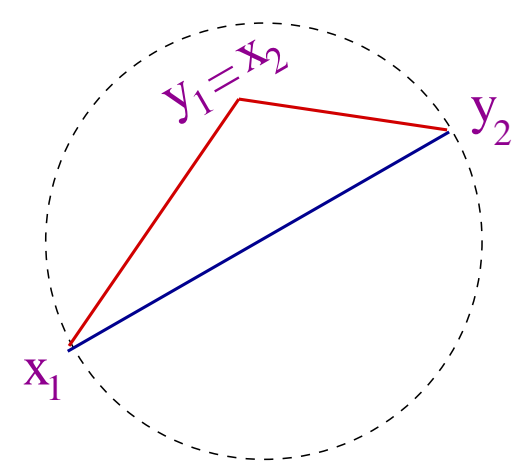

Fig. 1. The geometry of dipole splitting

It is in fact easy to turn some of the above considerations into more explicit formulae: The differential probability for a dipole $(\boldsymbol{x}, \boldsymbol{y})$ to split into new dipoles $(\boldsymbol{x}, \boldsymbol{z})$ and $(\boldsymbol{z}, \boldsymbol{y})$ is known within perturbative QCD (to leading-log accuracy with respect to $Y=\ln 1 / x)$ as $\left(\bar{\alpha}_{s} \equiv \alpha_{s} N_{c} / \pi\right)$

$$
d \mathcal{P}(\boldsymbol{x}, \boldsymbol{y} \mid \boldsymbol{z})=\frac{\bar{\alpha}_{s}}{2 \pi} \frac{(\boldsymbol{x}-\boldsymbol{y})^{2}}{(\boldsymbol{x}-\boldsymbol{z})^{2}(\boldsymbol{z}-\boldsymbol{y})^{2}} d^{2} \boldsymbol{z} d Y .
$$

This formula, together with elementary geometry considerations (see Fig. 1), immediately imply (with $\boldsymbol{r}_{1}=\boldsymbol{x}_{1}-\boldsymbol{y}_{1}$ and $\boldsymbol{r}_{2}=\boldsymbol{x}_{2}-\boldsymbol{y}_{2}$ ):

$$
\begin{array}{r}
\left.\frac{\partial}{\partial Y}\left\langle n^{(2)}\left(\boldsymbol{x}_{1}, \boldsymbol{y}_{1} ; \boldsymbol{x}_{2}, \boldsymbol{y}_{2}\right)\right\rangle_{Y}\right|_{\text {fluct. }}=\frac{\bar{\alpha}_{s}}{2 \pi} \frac{\left(\boldsymbol{r}_{1}+\boldsymbol{r}_{2}\right)^{2}}{\boldsymbol{r}_{1}^{2} \boldsymbol{r}_{2}^{2}}\left\{\delta^{(2)}\left(\boldsymbol{x}_{2}-\boldsymbol{y}_{1}\right)\left\langle n\left(\boldsymbol{x}_{1}, \boldsymbol{y}_{2}\right)\right\rangle_{Y}\right. \\
\left.+\delta^{(2)}\left(\boldsymbol{y}_{2}-\boldsymbol{x}_{1}\right)\left\langle n\left(\boldsymbol{x}_{2}, \boldsymbol{y}_{1}\right)\right\rangle_{Y}\right\} .(2.2
\end{array}
$$

As indicated in the l.h.s. of Eq. (2.2), this is just the "fluctuating" contribution to the evolution of $\left\langle n^{(2)}\right\rangle$, in which the two measured dipoles are generated via the splitting of a unique original dipole. The general equation for $\partial\left\langle n^{(2)}\right\rangle / \partial Y$, to be constructed in Sect. 5 within the dipole picture, involves also terms linear in $\left\langle n^{(2)}\right\rangle$, which correspond to the usual BFKL evolution. By also using the relation $T(\boldsymbol{r}, \boldsymbol{b}, Y) \sim \alpha_{s}^{2} r^{4} n(\boldsymbol{r}, \boldsymbol{b}, Y)$, between (target) dipole densities and (projectile) dipole scattering amplitudes, it is possible to transform evolution equations for the dipole densities, so like Eq. (2.2), into corresponding equations for the scattering amplitudes. This will be discussed in Sect. 6 .

\section{The Balitsky-JIMWLK equations and beyond}

Our discussion of the Balitsky-JIMWLK equations in this section will be rather schematic, as our intention is merely to show - by inspection of the structure of these equations, and with the help of a few Feynman diagrams - 
that these equations do not include the effects of gluon number fluctuations in the target wavefunction. Since, as we shall later argue, these fluctuations serve as the initiators of the "pomeron loops" in the target, our conclusion also implies that the evolution generated by these equations fails to include the pomeron loops. This failure was already recognized in the literature in relation with the Kovchegov equation, which is a mean field approximation to the Balitsky-JIMWLK equations, but to our knowledge it has not been argued so far for the general equations.

For consistency for the other developments in this paper, we shall restrict also the present discussion to the (high-energy version of the) large $-N_{c}$ limit, in which Balitsky-JIMWLK equations close in the space of dipoles. That is, they reduce to a hierarchy of equations for the evolution of the scattering amplitudes of a set of $N$ dipoles, with $N=1,2, \ldots$, which scatter off a generic target. The color fields in the target can be strong — that is, the target can be at saturation (a 'color glass condensate') —- but the equations include non-linear effects which ensure that scattering is unitary. As we shall see, the non-linear effects in the evolution can be interpreted as either multiple scattering, or saturation, depending upon the perspective from which one views the evolution (as projectile or, respectively, target evolution).

The simplest way to present the (dipolar version of the) Balitsky-JIMWLK equations is to notice that the whole hierarchy can be generated from the following "operator" equation:

$$
\begin{aligned}
\frac{\partial T_{Y}(\boldsymbol{x}, \boldsymbol{y})}{\partial Y}=\frac{\bar{\alpha}_{s}}{2 \pi} \int d^{2} \boldsymbol{z} \frac{(\boldsymbol{x}-\boldsymbol{y})^{2}}{(\boldsymbol{x}-\boldsymbol{z})^{2}(\boldsymbol{y}-\boldsymbol{z})^{2}} & \\
& \left\{-T_{Y}(\boldsymbol{x}, \boldsymbol{y})+T_{Y}(\boldsymbol{x}, \boldsymbol{z})+T_{Y}(\boldsymbol{z}, \boldsymbol{y})-T_{Y}(\boldsymbol{x}, \boldsymbol{z}) T_{Y}(\boldsymbol{z}, \boldsymbol{y})\right\}
\end{aligned}
$$

By "operator equation" we simply mean that, in order to deduce the equation satisfied by the $N$-point function $\left\langle T^{(N)}\right\rangle_{Y} \equiv\langle T(1) T(2) \cdots T(N)\rangle_{Y}$ (with $\left.T(i) \equiv T\left(\boldsymbol{x}_{i}, \boldsymbol{y}_{i}\right)\right)$, it is sufficient to multiply Eq. (3.1) by appropriate powers of $T$ and then use Leibniz' rule; e.g. :

$$
\frac{\partial}{\partial Y}\langle T(1) T(2)\rangle_{Y}=\left\langle\frac{\partial T(1)}{\partial Y} T(2)\right\rangle_{Y}+\left\langle T(1) \frac{\partial T(2)}{\partial Y}\right\rangle_{Y}
$$

In particular, the equation obeyed by the average scattering amplitude of a single dipole is immediately obtained as:

$$
\begin{aligned}
\frac{\partial}{\partial Y}\langle T(\boldsymbol{x}, \boldsymbol{y})\rangle_{Y}= & \frac{\bar{\alpha}_{s}}{2 \pi} \int d^{2} \boldsymbol{z} \frac{(\boldsymbol{x}-\boldsymbol{y})^{2}}{(\boldsymbol{x}-\boldsymbol{z})^{2}(\boldsymbol{y}-\boldsymbol{z})^{2}} \\
& \langle-T(\boldsymbol{x}, \boldsymbol{y})+T(\boldsymbol{x}, \boldsymbol{z})+T(\boldsymbol{z}, \boldsymbol{y})-T(\boldsymbol{x}, \boldsymbol{z}) T(\boldsymbol{z}, \boldsymbol{y})\rangle_{Y}
\end{aligned}
$$


As anticipated in Sect. 2, the r.h.s. of this equation involves the scattering amplitude $\left\langle T^{(2)}\right\rangle=\langle T(\boldsymbol{x}, \boldsymbol{z}) T(\boldsymbol{z}, \boldsymbol{y})\rangle$ for two contiguous dipoles. More generally, the equation for $\left\langle T^{(N)}\right\rangle_{Y}$ involves also $\left\langle T^{(N+1)}\right\rangle_{Y}$, so Eq. (3.1) generates an infinite hierarchy of equations which decouple from each other only in the weak scattering regime, where $\left\langle T^{(N+1)}\right\rangle \ll\left\langle T^{(N)}\right\rangle$ and each $N$-point function obeys separately the BFKL equation for evolution in any of its $N$ arguments.

But the hierarchy generated by Eq. (3.1) is sufficiently simple for the corresponding evolution to be quasi-deterministic. It is indeed easy to check that, if the initial conditions at $Y=Y_{0}$ are chosen in factorized form, i.e., $\langle T(1) \cdots T(N)\rangle_{0}=\langle T(1)\rangle_{0} \cdots\langle T(N)\rangle_{0}$, then this factorized form is preserved by the evolution up to arbitrarily large $Y$ : schematically, $\left\langle T^{(N)}\right\rangle_{Y}=\langle T\rangle_{Y}^{N}$ with the one-point function $\langle T\rangle_{Y}$ obeying the BK equation (i.e., the equation obtained by replacing $T \rightarrow\langle T\rangle_{Y}$ into Eq. (3.1)). More generally, it has been shown in Refs. [41,42] that the hierarchy generated by Eq. (3.1) admits a one-parameter family of fully factorized exact solutions. This strongly suggests that, in their simplified form valid at large $N_{c}$, the Balitsky-JIMWLK equations do not generate new correlations, but only propagate those already encoded in the initial conditions. This simplifying feature does not hold also for the full equations, which include additional, multipolar, operators. But the correlations induced by these operators are suppressed by powers of $1 / N_{c}^{2}$, and thus cannot be associated with fluctuations in the gluon (or dipole) number. Rather, as discussed in the Introduction, they describe color fluctuations.

This conclusion is further substantiated by an analysis of the diagrammatic content of the Balitsky-JIMWLK equations, to which we now turn. At this point one should recall that the structure of the perturbation theory, and thus the form of the diagrams, depends upon the frame in which one is viewing the evolution:

a) In the original derivation by Balitsky [7], the evolution is implemented by boosting the projectile, which then evolves through (small- $x$ ) gluon radiation. In the large $-N_{c}$ limit in which the projectile is a collection of $N$ dipoles, its evolution amounts to the splitting of one of these dipoles into two new dipoles, followed by the interaction between the final system of $N+1$ dipoles and the target. Then Eq. (3.1) applies to one such a dipole which has split, and the various terms there are easily interpreted: The two linear terms with positive sign, $T_{Y}(\boldsymbol{x}, \boldsymbol{z})$ and $T_{Y}(\boldsymbol{z}, \boldsymbol{y})$, describe the independent scattering of the daughter dipoles with the target, the quadratic term with a negative sign corrects for an overcounting of their simultaneous scattering, and the linear term with a negative sign is the "virtual term" which expresses the possibility that the parent dipole $(\boldsymbol{x}, \boldsymbol{y})$ survive without splitting.

In this picture, the non-linear terms in the evolution are thus associated with multiple scattering, but they indirectly reflect saturation effects in the 


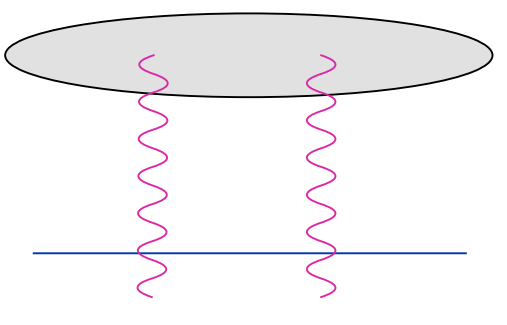

(a)

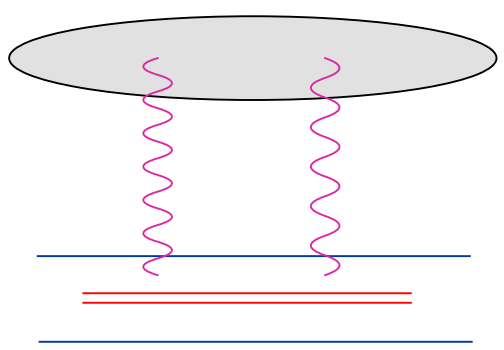

(c)

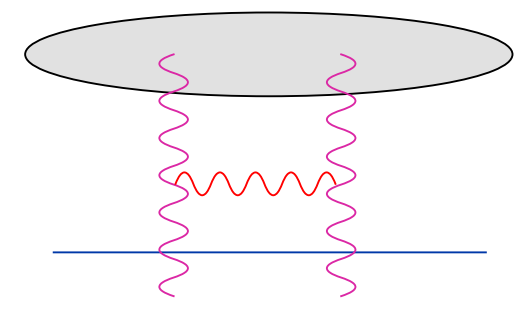

(e)

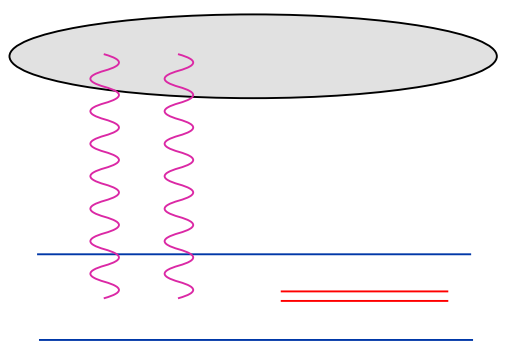

(b)

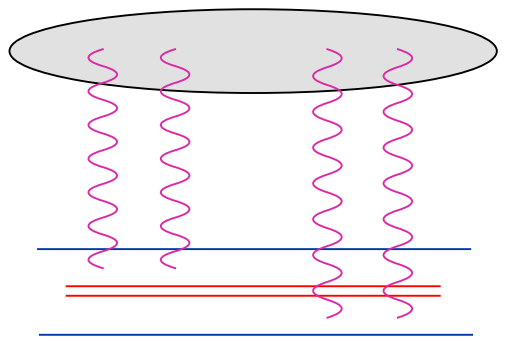

(d)

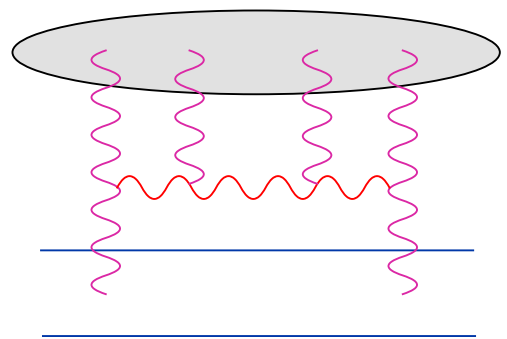

(f)

Fig. 2. Diagrams for single dipole scattering: (a) the tree-level contribution; $(b, c, d)$ one step evolution of the projectile; $(e, f)$ one step evolution of the target.

target. But as far as the projectile is concerned, there is still no saturation: The $N$ dipoles composing the projectile are not allowed to interact with each other, which is a good approximation only so long as $\alpha_{s}^{2} N \ll 1$. Since the total number of dipoles within the projectile grows exponentially with $Y$, this is clearly a low energy approximation.

In Fig. 2, we display a few Feynman diagrams which illustrate the processes encompassed by Eq. (3.1) from the perspective of projectile evolution. These diagrams are proper to the dipole picture, so the evolution is shown as dipole splitting rather than as gluon emission. For more clarity, we show only one diagram contributing to each type of process, which is moreover taken at the lowest non-trivial order in perturbation theory. Thus, the scattering between a dipole and the CGC target starts at two gluon exchange, as shown in Fig. 


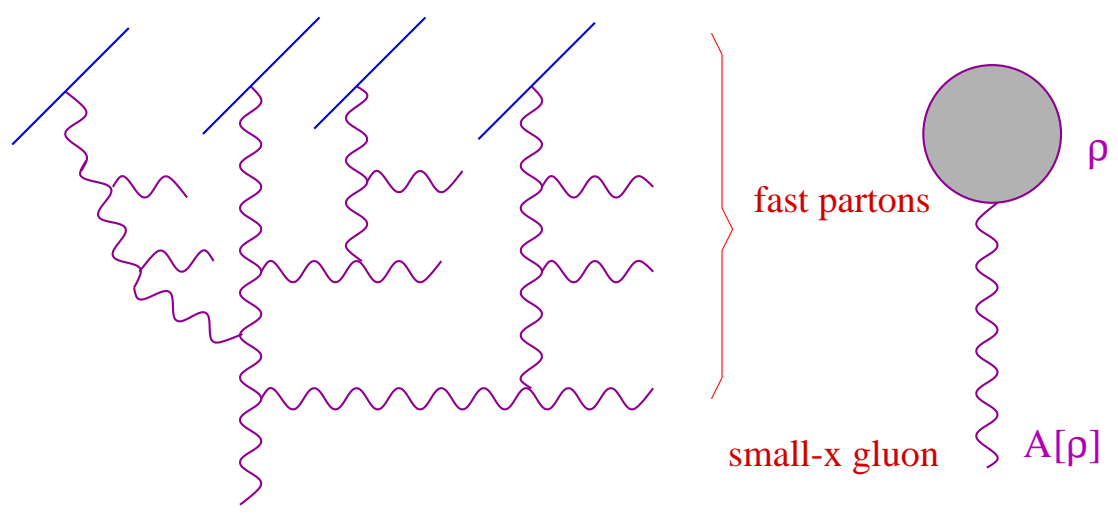

Fig. 3. A typical gluon cascade which contributes to a classical color field configuration in the color glass condensate

2.a. The one-step evolution of this amplitude then involves the diagrams in Figs. 2.b, c, d : Fig. 2.b is the virtual term (the original dipole interacts before splitting), Fig. 2.c shows the scattering of one of the daughter dipoles (there is a similar diagram for the other dipole), while Fig. 2.d describes their simultaneous scattering. We shall return to a discussion of these diagrams after also introducing the picture of the target evolution.

b) A different derivation of the Balitsky equations is provided [13] by the color glass condensate (CGC) formalism, in which the evolution is achieved by boosting the target, and the non-linear terms correspond explicitly to saturation effects in the target wavefunction. The 'color glass' is a random superposition of classical color fields with a gauge-invariant weight function which evolves with $Y$ according to JIMWLK equation $[12,13,16]$. Diagramatically, each classical field configuration corresponds to a set of gluon cascades which are initiated by 'color sources' (e.g., valence quarks) carrying a sizeable fraction of the target rapidity $Y$, and which end up with a small- $x$ gluon at the comparatively low rapidity of the projectile. (See Fig. 3 for a pictorial representation.) When increasing $Y$, these cascades evolve through gluon radiation from the classical field created in the previous steps. For weak fields ( $A \lesssim 1$ ), corresponding to low gluon density, the JIMWLK equation reduces to the standard BFKL evolution: the various $N$-point functions evolve independently from each other, and rise rapidly with $Y$. But at high density, the classical fields are strong $(A \gg 1$, and even $A \sim 1 / g$ in the 'condensate' regime at saturation), and the dynamics is fully non-linear.

For the subsequent discussion, it is important to notice that the nonlinearities in the CGC correspond to gluon merging, but not also to gluon splitting: As illustrated in Fig. 3, different gluon cascades can fuse with each other, via the non-linear effects encoded in the classical field equations (the Yang-Mills equations), and also in the emission and the propagation of the quantum gluons. This recombination process is what we refer to as 'gluon 
merging'. On the other hand, the quantum gluons radiated from the classical fields are not allowed to further radiate small $-x$ gluons by themselves; that is, the 'gluon splitting' is not included in the evolution. This amounts to neglecting gluon number fluctuations in the CGC picture. (Indeed, gluon splitting would be the CGC analog of the dipole splitting discussed in Sect. 2.) This is a good approximation at high density, where gluon splitting is indeed suppressed as compared to direct emission from the strong classical fields. But as we shall argue later, gluon splitting is a dominant effect in the dilute regime at high $k_{\perp}$, and plays also an essential role in the approach towards saturation.

This discussion has an obvious consequence for the structure of nonlinearities in the JIMWLK evolution equations for Green's functions: gluon merging can only reduce the number of active fields in a correlation function. Therefore, the r.h.s. of the equation for the $N$-point function involves in general all the $n$-point functions with $n \geq N$, but not also those with $n<N$ (compare in this respect with Eq. (2.2), where the opposite situation happens).

Consider now the scattering between the CGC and an external projectile which is a collection of dipoles. The scattering amplitude for a single dipole can be computed in the eikonal approximation as

$$
T(\boldsymbol{x}, \boldsymbol{y})=1-\frac{1}{N_{c}} \operatorname{tr}\left(V^{\dagger}(\boldsymbol{x}) V(\boldsymbol{y})\right)
$$

where $V^{\dagger}(\boldsymbol{x})$ and $V(\boldsymbol{y})$ are Wilson lines describing the scattering of the quark and, respectively, antiquark component of the dipole, e.g. (with $\mathrm{P}$ denoting path-ordering in $x^{-}$) :

$$
V^{\dagger}(\boldsymbol{x}) \equiv \mathrm{P} \exp \left(\mathrm{i} g \int d x^{-} A_{a}^{+}\left(x^{-}, \boldsymbol{x}\right) t^{a}\right)
$$

and $A_{a}^{+}$is the longitudinal component of the color field in the target (the only dynamical field left in the problem), whose correlations are described by the CGC. The evolution equation for $\langle T(1) T(2) \cdots T(N)\rangle_{Y}$ is then obtained by using the definition (3.4)-(3.5) of $T$ together with the equations satisfied by the field correlators $\left\langle A^{+}(1) A^{+}(2) \cdots\right\rangle_{Y}$, which in turn follow from the JIMWLK equation ${ }^{6}$. As anticipated, the ensuing equations are precisely those originally obtained by Balitsky, and which at large $N_{c}$ are generated by Eq. (3.1).

Let us now describe the diagrammatic interpretation of these equations from the perspective of target evolution. Once again, we show only a minimal set of representative diagrams. To easy read these diagrams, notice that the

\footnotetext{
${ }^{6}$ In practice, it is often simpler to work out directly the evolution equations for the correlations of the Wilson lines. But thinking in terms of field correlations helps clarifying the diagrammatic interpretation of the JIMWLK evolution.
} 
lowest-order contribution to the scattering amplitude (3.4), as obtained after expanding the Wilson lines there, is the 2-gluon exchange:

$$
T(\boldsymbol{x}, \boldsymbol{y}) \approx \frac{g^{2}}{4 N_{c}}\left(A_{a}^{+}(\boldsymbol{x})-A_{a}^{+}(\boldsymbol{y})\right)^{2}+\mathcal{O}\left(g^{3}\right)
$$

The two diagrams shown in Figs. 2.e and $f$ correspond to the linear and nonlinear terms in Eq. (3.1), respectively. The diagram in Fig. 2.e represents the first step in the BFKL evolution of the 2-point function $\left\langle A^{+} A^{+}\right\rangle_{Y}$ (the 'unintegrated gluon distribution'); in terms of projectile evolution, it corresponds to the diagrams shown in Figs. 2.b and c. But Fig. 2.f exhibits a non-linear effect which goes beyond BFKL evolution: four gluons merge into two (a 4-point function reduces to a 2-point function) through a vertex which at large $-N_{c}$ can be recognized ${ }^{7}$ as the 'triple pomeron vertex'. Fig. 2.f corresponds to Fig. 2.d for projectile evolution; both diagrams involve the same triple pomeron vertex, only with a different interpretation: $i$ ) In Fig. 2.d, this vertex describes the splitting of one dipole into two dipoles which then scatter both with the target (so that the net effect of the evolution is to replace a single-pomeron exchange, cf. Fig. 2.a, by a double-pomeron exchange, Fig. 2.d). ii) In Fig. 2.f, the same vertex describes gluon merging in the target wavefunction.

By 'iterating' the diagrams in Fig. 2, it is straightforward to deduce the diagrammatic interpretation of the equation satisfied by $\left\langle T^{(N)}\right\rangle_{Y}$ for any $N \geq 1$. Although this seems like a trivial exercise, we nevertheless exhibit the corresponding results for $\left\langle T^{(2)}\right\rangle_{Y}$ in Figs. 4.b and c (projectile evolution) and 4.d and e (target evolution), only to emphasize that these are not all the diagrams which would be expected within perturbative QCD to the order of interest. (Fig. 4.a shows the corresponding tree-level diagram.) This lack of perturbative completeness is to be related to our previous remarks concerning the lack of saturation effects in the projectile evolution and, respectively, the lack of gluon number fluctuations in the target evolution. As we shall shortly see, both omissions refer in fact to the same physical process, only seen from different points of view.

Specifically, in Figs. 4.f and g we show two diagrams which are formally of the same order in $\alpha_{s}$ as the diagrams for linear evolution in Figs. 4.b and $\mathrm{d}$, yet they were not included in the previous analysis. For the diagram in Fig. 4.f, this absence is easy to explain: this diagram is subleading at large $N_{c}$, so it is naturally missed by Eq. (3.1); as a matter of facts, this diagram is correctly included in the full version of Balitsky equations (valid for arbitrary

7 Indeed, according to Eq. (3.6), the four gluons which enter this vertex from the above are pairwise coupled into two color singlets, so like the two gluons emerging from the vertex; after subsequent evolution, any of these 2-gluon singlet exchanges would be eventually converted into BFKL ladders, or 'pomerons'. 


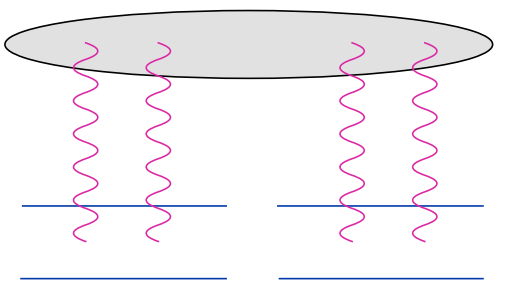

(a)

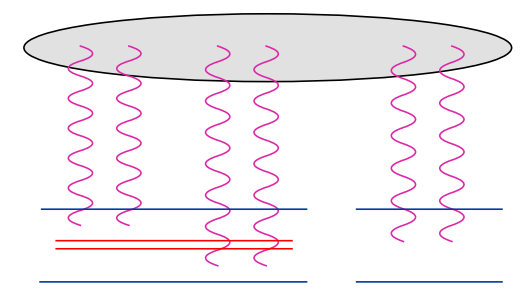

(c)

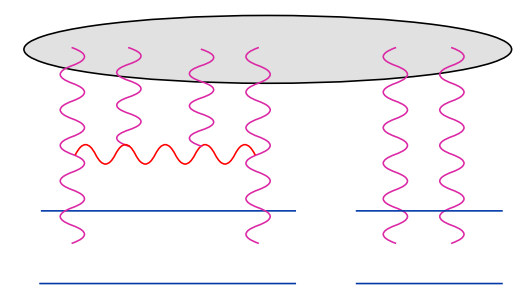

(e)

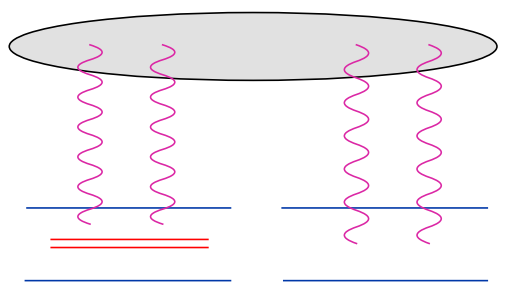

(b)

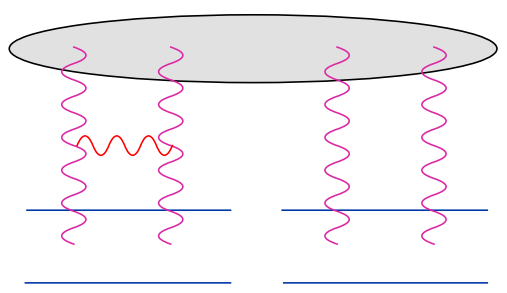

(d)

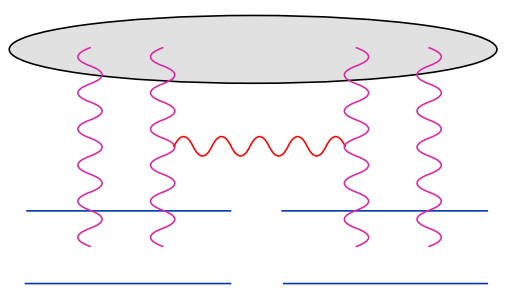

(f)

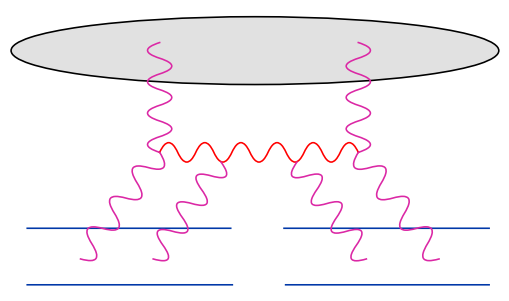

(g)

Fig. 4. Diagrams for dipole pair scattering: (a) tree-level contribution; (b, c) one step projectile evolution; ( $d$, e) one step target evolution; (f) a diagram suppressed at large $N_{c} ;(g)$ the missing diagram, which is of leading order in both $\alpha_{s}$ and $N_{c}$.

$N_{c}$ ), and also in the JIMWLK formalism. As for the second diagram, Fig. 4.g, this is easily recognized to describe the incriminated process, which is missing in either Balitsky, or JMWLK, picture of the non-linear evolution: Seen from the perspective of projectile evolution, this diagram describes the interaction between two dipoles in the projectile, that is, a saturation effect, whereas from the perspective of the target, it rather corresponds to gluon splitting.

By inspection of the diagrams in Fig. 4, one can understand what was 
the argument for ignoring such contributions in the JIMWLK evolution of the target, and also when is this argument expected to break down. Consider the dependence of the processes in Fig. 4 upon the gluon density in the target: A 2-point function counts like the density, a 4-point function, like the density squared, etc. Then the standard, BFKL, diagram in Fig. 4.d is proportional to the gluon density squared, the recombination diagram in Fig. 4.e scales like the third power of the density times an additional factor $\alpha_{s}$, while the splitting diagram, Fig. 4.g, scales likes $\alpha_{s}$ times the density ${ }^{8}$. Clearly, this last contribution is indeed negligible whenever the density is large enough. For instance, in the saturation region, the density is $\mathcal{O}\left(1 / \alpha_{s}\right)$, so both the linear diagram, Fig. 4.d, and the recombination diagram, Fig. 4.e, contribute on equal footing, whereas the splitting diagram, Fig. 4.g, is totally irrelevant. However, the situation changes in the dilute regime at high transverse momenta, where the density is very low (the average gluon occupation number can be much smaller than one); then, all diagrams are suppressed except for the splitting one, Fig. 4.g, which shows how a 4 -point correlation gets generated via the splitting of a 2-point one. Clearly, this is the dominant mechanism for constructing higher-point correlation functions in the dilute regime.

To summarize this discussion, the Balitsky-JIMWLK equations include a single type of triple pomeron vertex, which corresponds to either pomeron splitting, or pomeron merging, depending upon the perspective that we have over the evolution. Still, both merging and splitting are necessary in order to have a complete description of the approach towards saturation, which is consistent with perturbative QCD. Among the approaches to high energy evolution proposed so far, the dipole picture includes only splitting (and thus can be used to describe particle number fluctuations at high- $k_{\perp}$ ), whereas the JIMWLK evolution contains only merging (so it describes correctly the saturation effects at high density). As explained in the Introduction, our goal is to combine the respective virtues of these two approaches in order to achieve a more complete description of the high-energy evolution of the scattering amplitudes. Our strategy can be summarized as follows:

Since the physical regimes in which the interesting phenomena - saturation and fluctuations (or merging and splitting) — play important roles are kinematically well separated, it should be possible to use a 'piecewise' description in which each regime is covered by a different formalism. Such a description should correctly capture the essential physics that we are interested in, although it would probably fail to reproduce the fine details of the transition between the two regimes. Specifically, in what follows we shall stick

8 Overall, there is the same power of $\alpha_{s}$ associated with the diagrams in Figs. 4.d and 4.g. But when interpreting these diagrams in terms of gluon densities, one should remember that one factor of $\alpha_{s}$ is included in the definition of the gluon density. 
to target evolution, and we shall rely on the JIMWLK formalism in the highdensity regime, and on the dipole picture in the dilute one (which means, in particular, that we shall limit ourselves to the large $-N_{c}$ approximation). Thus, both gluon merging, Fig. 4.e, and gluon splitting, Fig. 4.g, will be encoded in the target wavefunction, although their respective theoretical descriptions will be different. But, as we shall see, these descriptions can be naturally merged with each other in the equations for the scattering amplitudes, thus leading to a generalization of the Balitsky-JIMWLK hierarchy which includes the effects of gluon number fluctuations in the dilute regime, and thus generates pomeron loops ${ }^{9}$ through iterations.

\section{A toy model borrowed from statistical physics}

A main difficulty in our present analysis is the lack of a unified theoretical framework for describing both saturation and particle number fluctuations in the hadron lightcone wavefunction. It is therefore instructive, before we embark ourselves in a more detailed study of QCD, to make a detour through a simple particle model borrowed from statistical physics which contains both physical ingredients (particle number fluctuations and recombination) in a unified setting. This model is formulated similarly to the QCD dipole picture that we shall discuss in the next section - there is a system of particles whose distribution evolves in time according to a master equation - but it goes beyond the dipole picture by including recombination effects (so, in that respect, it may be viewed as a non-linear version of the dipole picture).

For this model, we shall study the effects of fluctuations on the evolution equations for particle number correlations, and, in particular, we shall show that it is possible to exactly reformulate the dynamics as a specific Langevin equation. This is interesting since the Langevin equation is better suited for numerical studies than the original master equation. Inspired by this, we shall later be able to also reformulate the equations for scattering amplitudes in QCD as a corresponding Langevin equation. Besides, some of the conceptual and technical issues that we shall clarify in this section will appear again later, in the context of QCD.

For more clarity, in this section we shall consider the simplest version of the statistical model: a zero-dimensional system (no spatial dimension) in which the only interesting distribution is that of the particle number. In

9 The simplest 'pomeron loop' is a one-loop diagram generated by a (BFKL) pomeron which splits into two which then recombine with each other. Thus, for this diagram to exists, one needs the simultaneous presence of triple pomeron vertices for splitting and merging. 
the Appendix, we shall then discuss a more refined model (taken from Refs. $[38,43])$, which has one spatial dimension, and which in spite of its relative simplicity leads to a Langevin equation very similar to the one that we shall find in QCD. This is the sFKPP equation [39,40] (and Refs. therein).

Specifically, in this section we consider a system whose state is described by the number $n$ of particles A. One can think of all these particles as being located at some unique lattice site, in which case $n$ represents the occupation number of that site. The dynamics is determined by two random elementary processes: under an infinitesimal step $d Y$ in time, a particle can split into two with a rate $\alpha$ :

$$
\mathrm{A} \stackrel{\alpha}{\rightarrow} \mathrm{A}+\mathrm{A},
$$

while two particles can recombine into one with a rate $2 \beta$ :

$$
\mathrm{A}+\mathrm{A} \stackrel{2 \beta}{\longrightarrow} \mathrm{A}
$$

Clearly, the probability $P(n)=P(n, Y)$ to find a configuration with $n$ particles changes with time, and the corresponding master equation reads

$$
\frac{d P(n)}{d Y}=\left.\frac{d P(n)}{d Y}\right|_{\alpha}+\left.\frac{d P(n)}{d Y}\right|_{\beta},
$$

where the first term in the r.h.s. is the contribution of splitting:

$$
\left.\frac{d P(n)}{d Y}\right|_{\alpha}=\alpha[(n-1) P(n-1)-n P(n)],
$$

while the second one is due to recombination:

$$
\left.\frac{d P(n)}{d Y}\right|_{\beta}=\beta[n(n+1) P(n+1)-n(n-1) P(n)] .
$$

The expectation value of an observable $\mathcal{O}(n)$ is given by

$$
\langle\mathcal{O}(n)\rangle_{Y}=\sum_{n} P(n, Y) \mathcal{O}(n)
$$

and its evolution equation can be obtained in general from Eq. (4.3)-(4.5). For example, it is not hard to find how the average values of the "number operators" $\langle n\rangle,\left\langle n^{2}\right\rangle, \ldots$ change with time. We have

$$
\begin{aligned}
\frac{d\langle n\rangle}{d Y} & =\alpha\langle n\rangle-\beta\left[\left\langle n^{2}\right\rangle-\langle n\rangle\right] \\
\frac{d\left\langle n^{2}\right\rangle}{d Y} & =\alpha\left[2\left\langle n^{2}\right\rangle+\langle n\rangle\right]-\beta\left[\left\langle n^{3}\right\rangle-3\left\langle n^{2}\right\rangle+\langle n\rangle\right], \ldots
\end{aligned}
$$


We shall also need the normal-ordered number operators, defined as

$$
: n^{\kappa}:=n(n-1) \cdots(n-\kappa+1) .
$$

To understand why we call this a "normal-ordered product", imagine a secondquantized formalism in which particles of type $A$ are created, or annihilated, by standard Fock space operators, $a^{\dagger}$ and, respectively, $a$, with $\left[a^{\dagger}, a\right]=1$. Then the particle number operator is $n=a^{\dagger} a$, and we have $n^{2}=a^{\dagger} a a^{\dagger} a=$ $a^{\dagger} a^{\dagger} a a+a^{\dagger} a=: n^{2}:+n$, so $: n^{2}:=n(n-1)$, so like in Eq. (4.8). Note furthermore that $: n^{2}$ : gives zero when acting on a state with a single particle (unlike $n^{2}$, which yields one). Thus, in the present context, the normal-ordering in $: n^{2}$ : is introduced in order for this operator to properly count the pairs of particles (so that, e.g., a single particle cannot be counted like a pair), and similarly for $: n^{\kappa}:$. Moreover, as we shall see in the next section, and also in the Appendix, the use of normal ordering is the only way to avoid equal-point singularities for systems with spatial dimensions.

The evolution equation for $n^{(\kappa)} \equiv\left\langle: n^{\kappa}:\right\rangle$ takes a rather simple form:

$$
\frac{d n^{(\kappa)}}{d Y}=\alpha\left[\kappa n^{(\kappa)}+\kappa(\kappa-1) n^{(\kappa-1)}\right]-\beta\left[\kappa n^{(\kappa+1)}+\kappa(\kappa-1) n^{(\kappa)}\right] .
$$

But the simplicity is rather illusory, as $n^{(\kappa)}$ is coupled in the evolution with $n^{(\kappa-1)}$ and $n^{(\kappa+1)}$, so Eq. (4.9) is just a particular equation within an infinite hierarchy. In what follows, we shall study various aspects of this hierarchy: In particular, we shall discuss the respective roles of the multiplication and recombination processes, and also the relative importance of fluctuations versus mean field aspects at different stages of the evolution. But before doing that, let us first introduce a different stochastic process which is mathematically equivalent to the one that we have studied so far, in the sense of generating exactly the same correlations as obtained by solving the hierarchy in Eq. (4.9).

Specifically, let us consider the following Langevin equation

$$
\frac{d \tilde{n}}{d Y}=\alpha \tilde{n}-\beta \tilde{n}^{2}+\sqrt{2\left[\alpha \tilde{n}-\beta \tilde{n}^{2}\right]} \nu \equiv A+B \nu,
$$

where $\nu(Y)$ is a Gaussian white noise : $\langle\nu(Y)\rangle=0$ and $\left\langle\nu(Y) \nu\left(Y^{\prime}\right)\right\rangle=\delta(Y-$ $\left.Y^{\prime}\right)$, and the right hand side must be understood with the Ito prescription for time discretization (recall that a Langevin process is not differentiable): Namely, if one writes $Y=j \epsilon$, where $j=0,1,2, \ldots$ and $\epsilon$ is the length of the time step, then Eq. (4.10) should be properly understood as

$$
\frac{\tilde{n}_{j+1}-\tilde{n}_{j}}{\epsilon}=\alpha \tilde{n}_{j}-\beta \tilde{n}_{j}^{2}+\sqrt{2\left[\alpha \tilde{n}_{j}-\beta \tilde{n}_{j}^{2}\right]} \nu_{j+1} \equiv A_{j}+B_{j} \nu_{j+1},
$$

with 


$$
\left\langle\nu_{j}\right\rangle=0, \quad\left\langle\nu_{i} \nu_{j}\right\rangle=\frac{1}{\epsilon} \delta_{i j}
$$

which shows that updating the variables $\tilde{n}$ at time-step $j+1$ only requires knowledge of the variables at time-step $j$. The noise term in Eq. (4.10) is said to be multiplicative since it is multiplied by a function of $\tilde{n}$. It is easy to see that $\tilde{n}=0$ is an unstable fixed point for the evolution described by Eq. (4.10), whereas $\tilde{n}=\alpha / \beta$ is a stable fixed point, and corresponds to saturation. If the initial condition satisfies $0 \leq \tilde{n}(0) \leq \alpha / \beta$, this will remain true for all subsequent times.

For an arbitrary function $F(\tilde{n})$, Eq. (4.10) implies the following evolution equation

$$
\frac{d\langle F(\tilde{n})\rangle}{d Y}=\left\langle A F^{\prime}(\tilde{n})\right\rangle+\frac{1}{2}\left\langle B^{2} F^{\prime \prime}(\tilde{n})\right\rangle .
$$

By taking $F(\tilde{n})=\tilde{n}^{\kappa}$, it is straightforward to show that the hierarchy emerging from the Langevin problem is indeed equivalent to the hierarchy of the toy model, Eq. (4.9), provided we identify $\left\langle\tilde{n}^{\kappa}\right\rangle$ with $n^{(\kappa)}$. In particular, the noise term in Eq. (4.11) is responsible for the fluctuation terms in Eq. (4.9), i.e., the terms proportional to $\kappa(\kappa-1)$ within the square brackets.

It is in fact easy to check directly at the level of the original hierarchy, Eq. (4.9), that $n^{(\kappa)}=(\alpha / \beta)^{\kappa}$ is indeed a fixed point of the evolution. Moreover, in the presence of fluctuations, we do not expect other fixed points (see also below). Thus, for sufficiently large $Y$, the occupation number will saturate to $n_{\text {sat }}=\alpha / \beta$ independently of the initial conditions. We would like this saturation value to be large, $n_{\text {sat }} \gg 1$, in order for the analogy with QCD to be as close as possible. We shall therefore choose $\alpha \gg \beta$, which implies that the recombination effects (proportional to $\beta$ ) will be parametrically suppressed as compared to the growth effects (proportional to $\alpha$ ) except in the saturation regime - a situation similar to QCD. Thus, if one starts in a dilute regime $\left(n \ll n_{\text {sat }}\right)$ at $Y=0$, then it is possible to follow the evolution in the early stages (prior to saturation) by using the linearized version of Eq. (4.9), as obtained after neglecting recombination :

$$
\frac{d n^{(1)}}{d Y}=\alpha n^{(1)}, \quad \frac{d n^{(2)}}{d Y}=2 \alpha\left[n^{(2)}+n^{(1)}\right], \quad \ldots
$$

As we shall see in Sect. 5, the above equations are the analog of the equations satisfied by the dipole densities in the dipole picture. Note that these equations are still coupled with each other, because of the fluctuation terms: The term linear in $n^{(1)}$ in the r.h.s. of the evolution equation for $n^{(2)}$ describes a fluctuation in which one particle splits into two (so this is the analog of the dipole splitting term in Eq. (2.2)). One may expect fluctuations not to be important at large times where $n^{(2)} \gg n^{(1)}$, but in general this is not right. 
To see this, consider the solution to Eqs. (4.14) with generic initial conditions $n^{(1)}(0)=n_{0}$ and $n^{(2)}(0)=n_{0}^{(2)}$ :

$$
\begin{aligned}
& n^{(1)}(Y)=n_{0} \exp (\alpha Y), \\
& n^{(2)}(Y)=\left[n_{0}^{(2)}+2 n_{0}\right] \exp (2 \alpha Y)-2 n_{0} \exp (\alpha Y), \ldots
\end{aligned}
$$

An interesting initial condition is $n_{0}=1$ and $n_{0}^{(\kappa)}=0$ for $\kappa \geq 2$, meaning that there is a single particle in the initial state. (In QCD, this would correspond to the case where one starts the evolution with a single dipole, or, more generally, with an isolated fluctuation at high $k_{\perp}$.) Then Eqs. (4.15) show that so long as $\alpha Y \lesssim 1, n^{(1)}$ dominates over $n^{(2)}$ in the r.h.s. of the second equation (4.14), and thus is driving force for the growth in the number of pairs. In the absence of fluctuations, the pair number $n^{(2)}$ would remain zero for ever. For larger $Y$, $n^{(2)}$ starts to dominate over $n^{(1)}$ (as it increases faster), but the ratio

$$
\frac{n^{(2)}(Y)}{\left[n^{(1)}(Y)\right]^{2}}=2-2 \exp (-\alpha Y)
$$

approaches 2 , and thus differs from the naive prediction $n^{(2)}(Y) \approx\left[n^{(1)}(Y)\right]^{2}$ of the mean field approximation. In fact, one can check on Eqs. (4.15) that the only way to satisfy this mean-field factorization at intermediate values of $Y$ (prior to saturation) is to assume a large occupation number already in the initial conditions: $n_{0} \gg 1, n_{0}^{(2)} \approx n_{0}^{2} \gg n_{0}$, etc. (In QCD, this would correspond, e.g., to starting the evolution with a very large nucleus at $Y=0$.)

This conclusion is not altered by the addition of the recombination terms, which merely provide saturation: For the model at hand and for generic initial conditions $\left(n_{0} \sim \mathcal{O}(1)\right)$, the mean field approximation is justified only in the saturation regime achieved at very large $Y: Y \gtrsim Y_{0} \simeq(1 / \alpha) \ln (\alpha / \beta)$. For a more general system which involves also spatial dimensions, so like QCD or the reaction-diffusion model to be studied in the Appendix, particles can escape from the bulk, so there will be always a region in phase-space in which the density is low and the mean field approximation is not applicable, however large is $Y$ and independently of the initial conditions (even if one starts with a large nucleus): this is the tail of the distribution, in which the evolution is driven by fluctuations.

In view of the comparison with QCD, it is useful to notice that the analog of the (dipolar) Balitsky-JIMWLK equations in the present toy model are the equations obtained after neglecting the fluctuation terms in Eq. (4.9), that is

$$
\frac{d n^{(\kappa)}}{d Y}=\kappa\left[\alpha n^{(\kappa)}-\beta n^{(\kappa+1)}\right] \quad \text { (no fluctuation) }
$$

(Alternatively, the analog of Eq. (3.1) — the generating equation of the 
Balitsky-JIMWLK hierarchy — is the deterministic equation obtained after ignoring the noise term in the Langevin equation (4.10).) Neglecting fluctuations in the recombination term is indeed harmless, since this term is important only in the high-density regime, where fluctuations become irrelevant anyway. But for the growth term, which acts also in the dilute regime, the fluctuations are crucial, as shown by the previous analysis. In particular, the reduced hierarchy in Eq. (4.17) could never generate a high density system from an initial state which involves only one particle.

Another effect of the fluctuations is to wash out some remarkable properties of Balitsky equations, like the existence of exact factorized solutions [41,42] : Eq. (4.17) can be solved with the Ansatz $n^{(\kappa)}=c_{\kappa}\left[n^{(1)}\right]^{\kappa}$ provided the coefficients $c_{\kappa}$ satisfy $c_{\kappa}=c_{2}^{\kappa-1}$, in agreement with Ref. [42]. (In particular, $n^{(\kappa)}=\left(1 / c_{2}\right)(\alpha / \beta)^{\kappa}$ is a one-parameter family of fixed points for Balitsky equations.) But it can be easily checked that such a factorized solution does not exist for the complete equations including fluctuations, that is, Eq. (4.9).

More generally, we expect the fluctuations to wash out any sensitivity to the initial conditions after a sufficiently large evolution. For the zerodimensional model in this section, this is simply the statement that at large $Y$ the solution will converge to an unique fixed point, namely $n^{(\kappa)}=(\alpha / \beta)^{\kappa}$, independently of the initial state. For more complicated systems, which involve also spatial dimensions, we expect universality at late times (in the sense of insensitivity to the initial conditions) also for more complex aspects of the dynamics, like the behavior of high energy scattering amplitudes in QCD.

\section{Fluctuations and evolution in the dipole picture}

We now return to QCD with a discussion of particle number fluctuations in the dipole picture. To that aim, we shall not rely on the original formulation of the dipole picture due to Mueller $[5,6]$, but rather on its alternative formulation in Ref. [32] (see also [37]), which is better suited for a study of fluctuations. The main difference between these two formulations is that, loosely speaking, 'they put the evolution at different ends' (see also Figs. 5 and 6 below). More precisely, in Mueller's original formulation, the evolution proceeds via splitting at the highest rapidity end: the rapidity increment $d Y$ is used to accelerate the original dipole (the one which has initiated the evolution), which then undergoes an additional splitting, whose effects propagate in the whole configuration. But in this picture the correlation between the highrapidity dipole which has split and the low-rapidity one that we measure is distributed over many steps of rapidity, and thus is difficult to trace back. By contrast, in the formulation in Ref. [32], the splitting occurs in one of the

dipoles at the lowest rapidity end, which was itself generated in the previous 
step of the evolution, and which is the direct parent of the dipole we measure. This makes it easier to follow correlations associated with splitting, which are particularly important in the first few steps after the splitting occurs.

Following Ref. [32], the system of dipoles generated by the evolution up to rapidity $Y$ of an original dipole with coordinates $\boldsymbol{x}_{0}$ and $\boldsymbol{y}_{0}$ will be described as a stochastic ensemble of dipole configurations endowed with a probability law which evolves with $Y$ according to a master equation. Specifically, a given configuration is specified by the number of dipoles $N$ and by $N-1$ transverse coordinates $\left\{\boldsymbol{z}_{i}\right\}=\left\{\boldsymbol{z}_{1}, \boldsymbol{z}_{2}, \ldots \boldsymbol{z}_{N-1}\right\}$, such that the coordinates of the $N$ dipoles are $\left(\boldsymbol{z}_{0}, \boldsymbol{z}_{1}\right),\left(\boldsymbol{z}_{1}, \boldsymbol{z}_{2}\right), \ldots,\left(\boldsymbol{z}_{N-1}, \boldsymbol{z}_{N}\right)$, with $\boldsymbol{z}_{0} \equiv \boldsymbol{x}_{0}$ and $\boldsymbol{z}_{N} \equiv \boldsymbol{y}_{0}$. The probability $P_{N}\left(\left\{\boldsymbol{z}_{i}\right\} ; Y\right)$ to find a given configuration obeys the following evolution equation (this is similar to Eq. (4.4)) :

$$
\begin{aligned}
\frac{\partial P_{N}\left(\boldsymbol{z}_{1}, \ldots \boldsymbol{z}_{N-1} ; Y\right)}{\partial Y}= & -\frac{\bar{\alpha}_{s}}{2 \pi}\left[\sum_{i=1}^{N} \int d^{2} \boldsymbol{z} \mathcal{M}\left(\boldsymbol{z}_{i-1}, \boldsymbol{z}_{i}, \boldsymbol{z}\right)\right] P_{N}\left(\boldsymbol{z}_{1}, \ldots \boldsymbol{z}_{N-1} ; Y\right) \\
& +\frac{\bar{\alpha}_{s}}{2 \pi} \sum_{i=1}^{N-1} \mathcal{M}\left(\boldsymbol{z}_{i-1}, \boldsymbol{z}_{i+1}, \boldsymbol{z}_{i}\right) P_{N-1}\left(\boldsymbol{z}_{1}, \ldots, \boldsymbol{z}_{i-1}, \boldsymbol{z}_{i+1}, \ldots, \boldsymbol{z}_{N-1} ; Y\right)
\end{aligned}
$$

where we have defined the shorthand notation for the dipole kernel

$$
\mathcal{M}(\boldsymbol{x}, \boldsymbol{y}, \boldsymbol{z})=\frac{(\boldsymbol{x}-\boldsymbol{y})^{2}}{(\boldsymbol{x}-\boldsymbol{z})^{2}(\boldsymbol{y}-\boldsymbol{z})^{2}} .
$$

The expectation value of an operator $\mathcal{O}$ which depends only the dipole sizes is given by

$$
\langle\mathcal{O}(Y)\rangle=\sum_{N=1}^{\infty} \int d \Gamma_{N} P_{N}\left(\left\{\boldsymbol{z}_{i}\right\} ; Y\right) \mathcal{O}_{N}\left(\left\{\boldsymbol{z}_{i}\right\}\right)
$$

where the phase space integration is simply $d \Gamma_{N}=\mathrm{d}^{2} \boldsymbol{z}_{1} \mathrm{~d}^{2} \boldsymbol{z}_{2} \ldots \mathrm{d}^{2} \boldsymbol{z}_{N-1}$. Then by using the master equation (5.1) one can show that

$$
\begin{aligned}
\frac{\partial\langle\mathcal{O}(Y)\rangle}{\partial Y}=\frac{\bar{\alpha}_{s}}{2 \pi} \sum_{N=1}^{\infty} \int d \Gamma_{N} P_{N}\left(\left\{\boldsymbol{z}_{i}\right\} ; Y\right) \sum_{i=1}^{N} \int d^{2} \boldsymbol{z} \mathcal{M}\left(\boldsymbol{z}_{i-1}, \boldsymbol{z}_{i}, \boldsymbol{z}\right) \\
\times\left[-\mathcal{O}_{N}\left(\left\{\boldsymbol{z}_{i}\right\}\right)+\mathcal{O}_{N+1}\left(\left\{\boldsymbol{z}_{i}, \boldsymbol{z}\right\}\right)\right]
\end{aligned}
$$

where the $\boldsymbol{z}$ argument in $\mathcal{O}_{N+1}$ is to be placed between $\boldsymbol{z}_{i-1}$ and $\boldsymbol{z}_{i}$.

In what follows, we shall use Eq. (5.4) to derive evolution equations for the dipole number densities. Because of the difference alluded to before with respect to the original formulation of the dipole picture, the final equations that we shall obtain are not the same as the corresponding equations in the early literature on the dipole picture (see Refs. [5,6,30,31]), although the two sets of equations are mathematically equivalent (in the sense of providing identical results for identical initial conditions). Very recently, Levin and Lublinsky [8] 
have obtained the same equations that we shall derive here, but they used a more straightforward approach, based on a generating functional [37], which however hides some of the subtle points that we shall emphasize below, and which in our opinion are important to properly understand the results.

Consider first the average dipole number density. The corresponding operator for a $N$-dipole configuration is

$$
n_{N}(\boldsymbol{x}, \boldsymbol{y})=\sum_{j=1}^{N} \delta^{(2)}\left(\boldsymbol{z}_{j-1}-\boldsymbol{x}\right) \delta^{(2)}\left(\boldsymbol{z}_{j}-\boldsymbol{y}\right) .
$$

With $\mathcal{O}_{N} \equiv n_{N}$, the expression within the square bracket in the second line of Eq. (5.4) becomes

$$
\begin{aligned}
\Delta_{i}(\boldsymbol{x}, \boldsymbol{y}, \boldsymbol{z})= & -\delta^{(2)}\left(\boldsymbol{z}_{i-1}-\boldsymbol{x}\right) \delta^{(2)}\left(\boldsymbol{z}_{i}-\boldsymbol{y}\right) \\
& +\delta^{(2)}\left(\boldsymbol{z}_{i-1}-\boldsymbol{x}\right) \delta^{(2)}(\boldsymbol{z}-\boldsymbol{y})+\delta^{(2)}(\boldsymbol{z}-\boldsymbol{x}) \delta^{(2)}\left(\boldsymbol{z}_{i}-\boldsymbol{y}\right) .
\end{aligned}
$$

After simple manipulations we arrive at the following evolution equation for the average of the dipole number density $n_{Y}(\boldsymbol{x}, \boldsymbol{y}) \equiv\langle n(\boldsymbol{x}, \boldsymbol{y})\rangle_{Y}$ :

$$
\begin{aligned}
\frac{\partial n_{Y}(\boldsymbol{x}, \boldsymbol{y})}{\partial Y}= & \frac{\bar{\alpha}_{s}}{2 \pi} \int d^{2} \boldsymbol{z}\left[-\mathcal{M}(\boldsymbol{x}, \boldsymbol{y}, \boldsymbol{z}) n_{Y}(\boldsymbol{x}, \boldsymbol{y})\right. \\
& \left.+\mathcal{M}(\boldsymbol{x}, \boldsymbol{z}, \boldsymbol{y}) n_{Y}(\boldsymbol{x}, \boldsymbol{z})+\mathcal{M}(\boldsymbol{z}, \boldsymbol{y}, \boldsymbol{x}) n_{Y}(\boldsymbol{z}, \boldsymbol{y})\right] \\
\equiv & \frac{\bar{\alpha}_{s}}{2 \pi} \int d^{2} \boldsymbol{z} \mathcal{K}_{\boldsymbol{x} \boldsymbol{y} \boldsymbol{z}} \otimes n_{Y}(\boldsymbol{x}, \boldsymbol{y}) .
\end{aligned}
$$

As anticipated, this is not the same as the standard equation for the dipole number density in the dipole picture. In the latter, the splitting occurs in the original dipole $\left(\boldsymbol{x}_{0}, \boldsymbol{y}_{0}\right)$, so the more complete notation $n_{Y}\left(\boldsymbol{x}, \boldsymbol{y} \mid \boldsymbol{x}_{0}, \boldsymbol{y}_{0}\right)$ is needed for the dipole density. Then the standard equation reads

$$
\begin{aligned}
\frac{\partial n_{Y}\left(\boldsymbol{x}, \boldsymbol{y} \mid \boldsymbol{x}_{0}, \boldsymbol{y}_{0}\right)}{\partial Y}=\frac{\bar{\alpha}_{s}}{2 \pi} \int d^{2} \boldsymbol{z} \mathcal{M}\left(\boldsymbol{x}_{0}, \boldsymbol{y}_{0}, \boldsymbol{z}\right)\left[-n_{Y}\left(\boldsymbol{x}, \boldsymbol{y} \mid \boldsymbol{x}_{0}, \boldsymbol{y}_{0}\right)\right. \\
\left.+n_{Y}\left(\boldsymbol{x}, \boldsymbol{y} \mid \boldsymbol{x}_{0}, \boldsymbol{z}\right)+n_{Y}\left(\boldsymbol{x}, \boldsymbol{y} \mid \boldsymbol{z}, \boldsymbol{y}_{0}\right)\right]
\end{aligned}
$$

and is also recognized as the dipole version of the BFKL equation [1]. The two equations (5.7) and (5.8) are illustrated in Figs. 5 and 6, respectively. In spite of the formal differences, these equations are nevertheless equivalent ${ }^{10}$, as shown in Ref. [32].

Now we turn to the dipole pair density. As in the toy model considered in the previous section, the corresponding operator for a given $N$-dipole config-

$\overline{10}$ This can be checked by using the symmetry property $(\boldsymbol{x}-\boldsymbol{y})^{4} n_{Y}\left(\boldsymbol{x}, \boldsymbol{y} \mid \boldsymbol{x}_{0}, \boldsymbol{y}_{0}\right)=$ $\left(\boldsymbol{x}_{0}-\boldsymbol{y}_{0}\right)^{4} n_{Y}\left(\boldsymbol{x}_{0}, \boldsymbol{y}_{0} \mid \boldsymbol{x}, \boldsymbol{y}\right)$. 


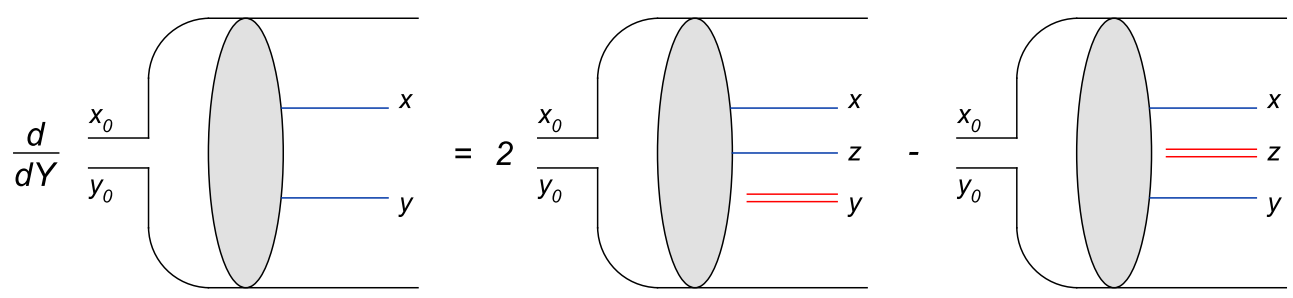

Fig. 5. The one-step evolution of the average dipole number density as described by Eq. (5.7).

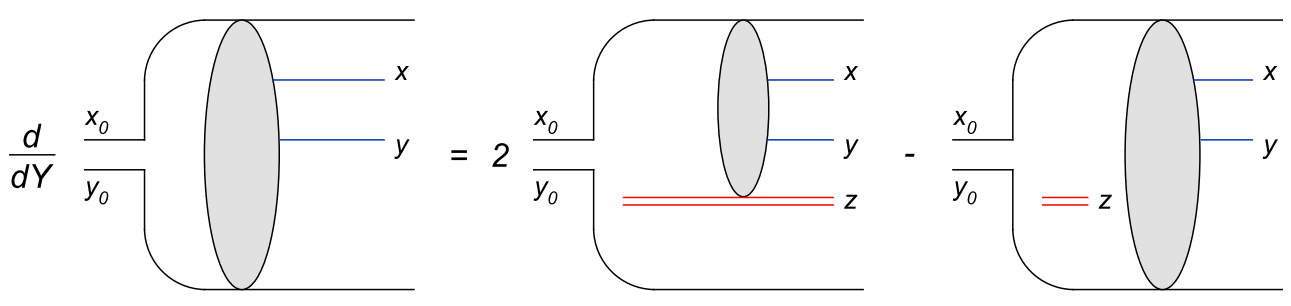

Fig. 6. The same as in Fig. 5, but from the perspective of Eq. (5.8).

uration is defined as the normal-ordered product of single densities:

$$
\begin{aligned}
n_{N}^{(2)}\left(\boldsymbol{x}_{1}, \boldsymbol{y}_{1} ; \boldsymbol{x}_{2}, \boldsymbol{y}_{2}\right) & =: n_{N}\left(\boldsymbol{x}_{1}, \boldsymbol{y}_{1}\right) n_{N}\left(\boldsymbol{x}_{2}, \boldsymbol{y}_{2}\right): \\
& \equiv n_{N}\left(\boldsymbol{x}_{1}, \boldsymbol{y}_{1}\right) n_{N}\left(\boldsymbol{x}_{2}, \boldsymbol{y}_{2}\right)-\delta^{(2)}\left(\boldsymbol{x}_{1}-\boldsymbol{x}_{2}\right) \delta^{(2)}\left(\boldsymbol{y}_{1}-\boldsymbol{y}_{2}\right) n_{N}\left(\boldsymbol{x}_{1}, \boldsymbol{y}_{1}\right)
\end{aligned}
$$

In fact, it is precisely this normal-ordered operator that is automatically reproduced by the generating functional used by Mueller [5,6], and also by Levin and Lublinsky $[8,37]$, but the importance of normal ordering has not been emphasized before, nor the difficulties arising when using the simple product (as opposed to the normal-ordered one) have been discussed.

The use of normal ordering is required by both physical and technical considerations $^{11}$. On the physical side, note that Eq. (5.9) is equivalent to a restricted double sum:

$n_{N}^{(2)}\left(\boldsymbol{x}_{1}, \boldsymbol{y}_{1} ; \boldsymbol{x}_{2}, \boldsymbol{y}_{2}\right)=\sum_{\substack{j, k=1 \\ j \neq k}}^{N} \delta^{(2)}\left(\boldsymbol{z}_{j-1}-\boldsymbol{x}_{1}\right) \delta^{(2)}\left(\boldsymbol{z}_{j}-\boldsymbol{y}_{1}\right) \delta^{(2)}\left(\boldsymbol{z}_{k-1}-\boldsymbol{x}_{2}\right) \delta^{(2)}\left(\boldsymbol{z}_{k}-\boldsymbol{y}_{2}\right)$,

which does not allow for "pairs" made of the same dipole. On the technical side, note that the subtraction performed in the second line of Eq. (5.9) eliminates the 'ultraviolet' singularity of the simple product $n_{N}\left(\boldsymbol{x}_{1}, \boldsymbol{y}_{1}\right) n_{N}\left(\boldsymbol{x}_{2}, \boldsymbol{y}_{2}\right)$ in the limit where the two dipoles have identical coordinates ${ }^{12}$. Because of

${ }^{11}$ We are grateful to Larry McLerran for his insightful observations on the role of normal ordering in this particular context.

12 This is manifest on the expression in Eq. (5.10) which is well defined when $\boldsymbol{x}_{1}=\boldsymbol{x}_{2}$ and $\boldsymbol{y}_{1}=\boldsymbol{y}_{2}$. The only dangerous term in this limit would have been the term with 
that, the evolution equation for the normal-ordered pair density is well defined in the continuum limit, unlike for the simple product.

Specifically, by using Eq. (5.9), we see that the square bracket in the second line of Eq. (5.4) becomes (recall also Eq. (5.6))

$$
\begin{aligned}
n_{N+1}^{(2)}-n_{N}^{(2)} & =\Delta_{i}\left(\boldsymbol{x}_{1}, \boldsymbol{y}_{1}, \boldsymbol{z}\right) n_{N}\left(\boldsymbol{x}_{2}, \boldsymbol{y}_{2}\right)+\Delta_{i}\left(\boldsymbol{x}_{2}, \boldsymbol{y}_{2}, \boldsymbol{z}\right) n_{N}\left(\boldsymbol{x}_{1}, \boldsymbol{y}_{1}\right) \\
& +\Delta_{i}\left(\boldsymbol{x}_{1}, \boldsymbol{y}_{1}, \boldsymbol{z}\right) \Delta_{i}\left(\boldsymbol{x}_{2}, \boldsymbol{y}_{2}, \boldsymbol{z}\right)-\delta^{(2)}\left(\boldsymbol{x}_{1}-\boldsymbol{x}_{2}\right) \delta^{(2)}\left(\boldsymbol{y}_{1}-\boldsymbol{y}_{2}\right) \Delta_{i}\left(\boldsymbol{x}_{1}, \boldsymbol{y}_{1}, \boldsymbol{z}\right)
\end{aligned}
$$

The contribution of the first two terms to the evolution equation is rather easy to obtain and reads (to simplify writing, we use the notation introduced in Eq. (5.7) together with $n_{Y}^{(2)} \equiv\left\langle n^{(2)}\right\rangle_{Y}$ )

$$
\frac{\bar{\alpha}_{s}}{2 \pi} \int d^{2} \boldsymbol{z}\left[\mathcal{K}_{\boldsymbol{x}_{1} \boldsymbol{y}_{1} \boldsymbol{z}}+\mathcal{K}_{\boldsymbol{x}_{2} \boldsymbol{y}_{2} \boldsymbol{z}}\right] \otimes n_{Y}^{(2)}\left(\boldsymbol{x}_{1}, \boldsymbol{y}_{1} ; \boldsymbol{x}_{2}, \boldsymbol{y}_{2}\right)+\text { linear }
$$

where the linear terms not explicitly shown arise from normal-ordering the product $n_{N}\left(\boldsymbol{x}_{1}, \boldsymbol{y}_{1}\right) n_{N}\left(\boldsymbol{x}_{2}, \boldsymbol{y}_{2}\right)$. By themselves, these linear terms are divergent when $\boldsymbol{x}_{1}=\boldsymbol{x}_{2}$ and/or $\boldsymbol{y}_{1}=\boldsymbol{y}_{2}$, but in the complete calculation they exactly cancel the singular terms coming from $\Delta_{i}\left(\boldsymbol{x}_{1}, \boldsymbol{y}_{1}, \boldsymbol{z}\right) \Delta_{i}\left(\boldsymbol{x}_{2}, \boldsymbol{y}_{2}, \boldsymbol{z}\right)$ and from the last term in Eq. (5.11). All the remaining terms are non-singular, and add up to give the following contribution to $n_{N+1}^{(2)}-n_{N}^{(2)}$ :

$$
\left[\delta^{(2)}\left(\boldsymbol{z}_{i-1}-\boldsymbol{x}_{1}\right) \delta^{(2)}\left(\boldsymbol{z}-\boldsymbol{y}_{1}\right) \delta^{(2)}\left(\boldsymbol{z}-\boldsymbol{x}_{2}\right) \delta^{(2)}\left(\boldsymbol{z}_{i}-\boldsymbol{y}_{2}\right)\right]+\{1 \leftrightarrow 2\},
$$

which in turn gives the following contribution, linear in the dipole number density $n_{Y}$, to the evolution equation for the dipole pair density :

$$
\frac{\bar{\alpha}_{s}}{2 \pi} \mathcal{M}\left(\boldsymbol{x}_{1}, \boldsymbol{y}_{2}, \boldsymbol{x}_{2}\right) n_{Y}\left(\boldsymbol{x}_{1}, \boldsymbol{y}_{2}\right) \delta^{(2)}\left(\boldsymbol{x}_{2}-\boldsymbol{y}_{1}\right)+\{1 \leftrightarrow 2\} .
$$

Putting everything together we finally arrive at

$$
\begin{aligned}
& \frac{\partial n_{Y}^{(2)}\left(\boldsymbol{x}_{1}, \boldsymbol{y}_{1} ; \boldsymbol{x}_{2}, \boldsymbol{y}_{2}\right)}{\partial Y}=\frac{\bar{\alpha}_{s}}{2 \pi}\left[\int d^{2} \boldsymbol{z} \mathcal{K}_{\boldsymbol{x}_{1} \boldsymbol{y}_{1} \boldsymbol{z}} \otimes n_{Y}^{(2)}\left(\boldsymbol{x}_{1}, \boldsymbol{y}_{1} ; \boldsymbol{x}_{2}, \boldsymbol{y}_{2}\right)\right. \\
& \left.+\mathcal{M}\left(\boldsymbol{x}_{1}, \boldsymbol{y}_{2}, \boldsymbol{x}_{2}\right) n_{Y}\left(\boldsymbol{x}_{1}, \boldsymbol{y}_{2}\right) \delta^{(2)}\left(\boldsymbol{x}_{2}-\boldsymbol{y}_{1}\right)\right]+\{1 \leftrightarrow 2\} .
\end{aligned}
$$

The terms proportional to $n_{Y}^{(2)}$ in the r.h.s. of the above equation describe the normal BFKL evolution of the pair density, while the terms linear in $n_{Y}$ are recognized as fluctuations in which the two measured dipoles arise from the splitting of the same parent dipole (and thus are contiguous with each other). These are precisely the fluctuations discussed in Sect. 2. As explained there, and also for the statistical model in Sect. 4, the main effect of these fluctuations is to drive the growth of pair density in the dilute regime where $n_{Y}$ dominates over $n_{Y}^{(2)}$ in the r.h.s. of Eq. (5.15).

$\overline{j=k \text { in }}$ the unrestricted double sum. 


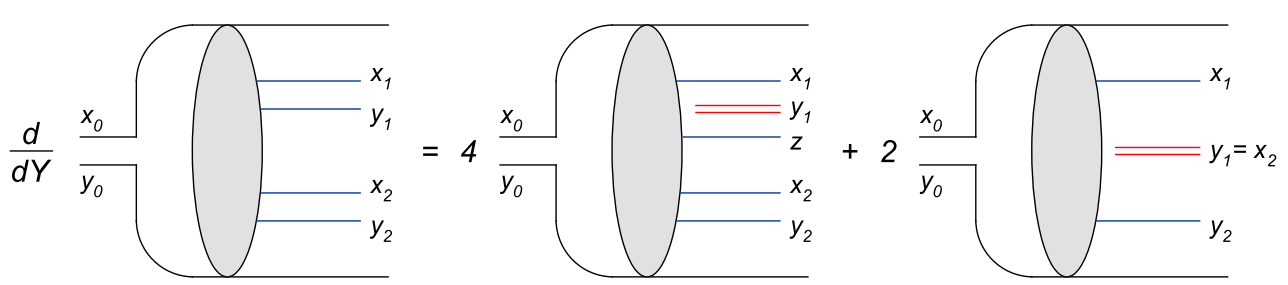

Fig. 7. The one-step evolution of the dipole pair density $n_{Y}^{(2)}$ as described by Eq. (5.15). The virtual BFKL term is not shown.

The evolution described by Eq. (5.15) is illustrated in Fig. 7. Once again, this equation is not the same as the standard equation for $n_{Y}^{(2)}$ which appears in the literature $[5,6,30,31]$ (but it coincides with the corresponding equation of Levin and Lublinsky [8]). The standard equation is rather obtained by putting the evolution in the original dipole (so like in Eq. (5.8)), and its r.h.s. involves $n_{Y}^{(2)}$ together with terms quadratic in $n_{Y}$, but not also terms linear in $n_{Y}$. Fluctuations are therefore more difficult to recognize on that equation.

For later use it is also convenient to express the fluctuation term in terms of the dipole sizes $\boldsymbol{r}_{i}=\boldsymbol{x}_{i}-\boldsymbol{y}_{i}$ and the corresponding impact parameters $\boldsymbol{b}_{i}=\left(\boldsymbol{x}_{i}+\boldsymbol{y}_{i}\right) / 2$, instead of the coordinates of their quark and anti-quark legs. One thus finds

$$
\frac{\bar{\alpha}_{s}}{2 \pi} \frac{\left(\boldsymbol{r}_{1}+\boldsymbol{r}_{2}\right)^{2}}{\boldsymbol{r}_{1}^{2} \boldsymbol{r}_{2}^{2}} n_{Y}\left(\boldsymbol{r}_{1}+\boldsymbol{r}_{2}, \boldsymbol{b}_{1}-\frac{\boldsymbol{r}_{2}}{2}\right) \delta^{(2)}\left(\boldsymbol{b}_{1}-\boldsymbol{b}_{2}-\frac{\boldsymbol{r}_{1}+\boldsymbol{r}_{2}}{2}\right)+\{1 \leftrightarrow 2\},
$$

which is the same as Eq. (2.2), as expected.

Note finally that via the same manipulations as above it is straightforward to construct the equation obeyed by the $k$-body dipole density $n_{Y}^{(k)}$ within the dipole picture. This is the analog of the $\alpha$-piece of Eq. (4.9) in the statistical

model, that is, it involves a piece proportional to $n_{Y}^{(k)}$ which represents the normal BFKL evolution, and a piece linear in $n_{Y}^{(k-1)}$ which describes fluctuations and dominates at low density. The corresponding equations can be found in Ref. [8].

\section{Fluctuations and saturation in dipole-CGC scattering}

In the previous section, we have seen that the dipole picture provides a natural theoretical framework to describe gluon number fluctuations in the dilute regime, and for large $N_{c}$. In what follows, we shall exploit the relation between dipole densities and scattering amplitudes in order to transfer the effects of fluctuations from the target wavefunction to the evolution equations for the amplitudes. In the dilute regime, where the fluctuations are important, 
the scattering amplitudes are simply proportional to the dipole densities in the target (see Sect. 6.1), and it is therefore straightforward to translate the previous equations in Sect. 5 into corresponding equations for the amplitudes. The ensuing equations can be then extended to the high-density regime by simply adding the same non-linear terms as in the Balitsky-JIMWLK equations (since the fluctuations cannot change significantly the non-linear aspects of the dynamics). These manipulations will lead us, in Sect. 6.2, to a new set of equations which include both fluctuations and saturation, and which are the main result in this paper. Finally, in Sect. 6.3, we shall show that, mediating a coarse-graining in impact parameter space, this infinite set of equations can be replaced by a single Langevin equation, which looks formally like the Kovchegov equation supplemented by a noise term.

\subsection{From dipole densities to scattering amplitudes}

Let us start with the scattering between a single external dipole ("the projectile") and a dilute target whose wavefunction is described in the dipole picture. The very statement that "the target is dilute" does not refer to the target alone, but rather is a statement about how the target looks like on the resolution scale of the projectile, which is its transverse size. Namely, this implies that the size $r$ of the external dipole is small as compared to the local saturation length $1 / Q_{s}(Y, \boldsymbol{b})$ in the target at the dipole impact parameter $\boldsymbol{b}$. Under these conditions, the scattering with the external dipole acts as a measure of the density of gluons (or dipoles) with transverse size $r$ in the target wavefunction. This is so since the dipole-dipole scattering is quasi-local in transverse phase-space, as we recall now:

A priori, the external dipole can scatter off any of the dipoles present in the target wavefunction, so its scattering amplitude can be evaluated as :

$$
T(\boldsymbol{r}, \boldsymbol{b}, Y)=\int d^{2} \boldsymbol{r}_{1} \int d^{2} \boldsymbol{b}_{1} \mathcal{T}_{0}\left(\boldsymbol{r}, \boldsymbol{r}_{1}, \boldsymbol{b}-\boldsymbol{b}_{1}\right) n\left(\boldsymbol{r}_{1}, \boldsymbol{b}_{1}, Y\right)
$$

where $n\left(\boldsymbol{r}_{1}, \boldsymbol{b}_{1}, Y\right)$ is the number density of dipoles with size $\boldsymbol{r}_{1}$ and impact parameter $\boldsymbol{b}_{1}$ in the target wavefunction, as produced after a rapidity evolution $Y$, and $\mathcal{T}_{0}\left(\boldsymbol{r}, \boldsymbol{r}_{1}, \boldsymbol{b}-\boldsymbol{b}_{1}\right)$ is the amplitude for the scattering between two elementary dipoles. Eq. (6.1) holds for a given configuration of the target wavefunction, that is, for a given scattering event. The corresponding expression for the average amplitude is obtained after averaging over all the configurations in the target, as explained in Sect. 5.

Note that in writing Eq. (6.1) we have restricted ourselves to a singlescattering approximation, as appropriate in the dilute regime. This equation 
is correct only so long as $T(\boldsymbol{r}, \boldsymbol{b}, Y) \ll 1$. For consistency, it is sufficient to evaluate $\mathcal{T}_{0}$ to lowest order in perturbation theory, that is, to $\mathcal{O}\left(\alpha_{s}^{2}\right)$, which corresponds to the exchange of two gluons. The corresponding expression is well known in the literature, but here we shall need only its general properties. Namely, $\mathcal{T}_{0} \sim \alpha_{s}^{2}$ when the two dipoles have similar sizes and are relatively close to each other in impact parameter space, but it decreases very fast, as $\mathcal{T}_{0}\left(\boldsymbol{r}, \boldsymbol{r}_{1}, \boldsymbol{b}-\boldsymbol{b}_{1}\right) \sim \alpha_{s}^{2} r^{2} r_{1}^{2} /\left(\boldsymbol{b}-\boldsymbol{b}_{1}\right)^{4}$, for a large separation $\left|\boldsymbol{b}-\boldsymbol{b}_{1}\right| \gg r_{>}$ between the two dipoles (with $r_{>}=\max \left(r, r_{1}\right)$ ). One can thus replace $\boldsymbol{b}_{1} \rightarrow \boldsymbol{b}$ as the argument of the density $n$ in the r.h.s. of Eq. (6.1), and at the same time evaluate the integral over $\boldsymbol{b}_{1}$ as:

$$
\int d^{2} \boldsymbol{b}_{1} \mathcal{T}_{0}\left(\boldsymbol{r}, \boldsymbol{r}_{1}, \boldsymbol{b}-\boldsymbol{b}_{1}\right) \simeq \int_{r_{>}} d^{2} \boldsymbol{b}_{r} \frac{\alpha_{s}^{2} r^{2} r_{1}^{2}}{\boldsymbol{b}_{r}^{4}} \simeq \alpha_{s}^{2} r_{<}^{2},
$$

where $\boldsymbol{b}_{r}=\boldsymbol{b}-\boldsymbol{b}_{1}$ and $r_{<}=\min \left(r, r_{1}\right)$.

To evaluate also the remaining integral over $\boldsymbol{r}_{1}$, one needs to know the corresponding dependence of the dipole density. So long as we are in the genuine BFKL regime, characterized by a non-trivial "anomalous dimension", the dominant behavior of $n\left(\boldsymbol{r}_{1}, \boldsymbol{b}, Y\right)$ is expected to be of the form

$$
n\left(\boldsymbol{r}_{1}, \boldsymbol{b}, Y\right) \sim \frac{1}{\boldsymbol{r}_{1}^{4}}\left(\boldsymbol{r}_{1}^{2} Q_{c}^{2}(\boldsymbol{b}, Y)\right)^{\gamma_{0}}
$$

where $\gamma_{0} \approx 0.63,1-\gamma_{0}$ is the anomalous dimension, and $Q_{c}^{2}(\boldsymbol{b}, Y) \propto \mathrm{e}^{\lambda \bar{\alpha}_{s} Y}$ is a line of constant density (up to the kinematical factor $\left.1 / \boldsymbol{r}_{1}^{4}\right)$ in the $\ln \left(1 / r_{1}\right)-Y$ plane. (This line is parallel to the saturation line.) Strictly speaking, Eq. (6.3) holds for the average density, but for sufficiently large $Y$, we expect the dipole distribution to self-average even in a single event, and therefore show the same gross features as the average distribution. After inserting Eq. (6.3) in the r.h.s. of Eq. (6.1), one can easily check that the ensuing integral is saturated by $r_{1} \sim r$, so, by dimensional arguments, the final result reads simply:

$$
T(\boldsymbol{r}, \boldsymbol{b}, Y) \simeq \alpha_{s}^{2} r^{4} n(\boldsymbol{r}, \boldsymbol{b}, Y)
$$

In the dilute BFKL regime in which we are interested here, this equation is exact up to some numerical fudge factor of order one, that we do not control in the above approximation, and which should depend upon the value of the anomalous dimension.

Eq. (6.4) can be immediately extended to the scattering of two or more external dipoles. In a particular event, the scattering amplitude for a system of several external dipoles is simply the product of the individual amplitudes for each dipole. For instance, 


$$
\begin{aligned}
T_{Y}^{(2)}\left(\boldsymbol{r}_{1}, \boldsymbol{b}_{1} ; \boldsymbol{r}_{2}, \boldsymbol{b}_{2}\right) & =T\left(\boldsymbol{r}_{1}, \boldsymbol{b}_{1}, Y\right) T\left(\boldsymbol{r}_{2}, \boldsymbol{b}_{2}, Y\right) \\
& \simeq \alpha_{s}^{4} r_{1}^{4} r_{2}^{4}: n\left(\boldsymbol{r}_{1}, \boldsymbol{b}_{1}, Y\right) n\left(\boldsymbol{r}_{2}, \boldsymbol{b}_{2}, Y\right):,
\end{aligned}
$$

where the final expression is correct up to some unknown fudge factor. Note that this final expression involves the normal-ordered dipole pair density, as defined in Eqs. (5.9)-(5.10). This is so since we neglect here the possibility that both external dipoles scatter off the same dipole in the target. As explained in Sect. 2, this is a good approximation so long as the external dipoles have different sizes and/or impact parameters.

\subsection{The equations for the scattering amplitudes}

By using Eqs. (6.4) and (6.5) together with the equations for the average one-body and two-body dipole densities in the target, as established in Sect. 5 , one can immediately deduce the equations satisfied by the average scattering amplitudes within the present approximations.

For the scattering amplitude of a single dipole $\langle T(\boldsymbol{r}, \boldsymbol{b})\rangle_{Y} \equiv\langle T(\boldsymbol{x}, \boldsymbol{y})\rangle_{Y}$, we thus find the BFKL equation, as expected:

$$
\begin{aligned}
\frac{\partial\langle T(\boldsymbol{x}, \boldsymbol{y})\rangle_{Y}}{\partial Y} & =\frac{\bar{\alpha}_{s}}{2 \pi} \int d^{2} \boldsymbol{z} \mathcal{M}(\boldsymbol{x}, \boldsymbol{y}, \boldsymbol{z})\langle-T(\boldsymbol{x}, \boldsymbol{y})+T(\boldsymbol{x}, \boldsymbol{z})+T(\boldsymbol{z}, \boldsymbol{y})\rangle_{Y} \\
& \equiv \frac{\bar{\alpha}_{s}}{2 \pi} \int d^{2} \boldsymbol{z} \mathcal{M}_{\boldsymbol{x} \boldsymbol{y} \boldsymbol{z}} \otimes\langle T(\boldsymbol{x}, \boldsymbol{y})\rangle_{Y}
\end{aligned}
$$

Note that after multiplication by the fourth power of the dipole size, the various dipole kernels appearing in the original equation (5.7) for the dipole density have been all converted into the same kernel $\mathcal{M}(\boldsymbol{x}, \boldsymbol{y}, \boldsymbol{z})$.

Eq. (6.6) applies so long as $T \ll 1$. But we know already what should be the corresponding generalization to the high density regime where $T \sim 1$ : In that regime, the target wavefunction is described by the CGC formalism, which should not be significantly altered by fluctuations, because the latter are relatively unimportant when the density is large. Thus, the general equation for $\langle T\rangle$ can be simply obtained by adding to Eq. (6.6) the appropriate nonlinear term generated by the JIMWLK evolution of the target. As discussed in Sect. 3, this non-linear term is $\langle-T(\boldsymbol{x}, \boldsymbol{z}) T(\boldsymbol{z}, \boldsymbol{y})\rangle_{Y}$. With this addition, Eq. (6.6) becomes identical to the first equation (3.3) in the Balitsky hierarchy.

Consider now the scattering amplitude for two dipoles $\left\langle T^{(2)}\left(\boldsymbol{x}_{1}, \boldsymbol{y}_{1} ; \boldsymbol{x}_{2}, \boldsymbol{y}_{2}\right)\right\rangle_{Y}$ $\equiv\left\langle T\left(\boldsymbol{x}_{1}, \boldsymbol{y}_{1}\right) T\left(\boldsymbol{x}_{2}, \boldsymbol{y}_{2}\right)\right\rangle_{Y}$. In the dilute regime, this is obtained by combining Eqs. (6.5) and (5.15), while in the high density regime this should also include the non-linear terms (involving $\left\langle T^{(3)}\right\rangle_{Y}$ ) induced by the JIMWLK evolution. 
By putting all these ingredients together, we find the following evolution equation (with the concise notation introduced in the second line of Eq. (6.6))

$$
\begin{aligned}
\frac{\partial\left\langle T^{(2)}\left(\boldsymbol{x}_{1}, \boldsymbol{y}_{1} ; \boldsymbol{x}_{2}, \boldsymbol{y}_{2}\right)\right\rangle_{Y}}{\partial Y}=\frac{\bar{\alpha}_{s}}{2 \pi}\{ & \int d^{2} \boldsymbol{z}\left(\mathcal{M}_{\boldsymbol{x}_{1} \boldsymbol{y}_{1} \boldsymbol{z}} \otimes\left\langle T^{(2)}\left(\boldsymbol{x}_{1}, \boldsymbol{y}_{1} ; \boldsymbol{x}_{2}, \boldsymbol{y}_{2}\right)\right\rangle_{Y}\right. \\
& \left.-\mathcal{M}\left(\boldsymbol{x}_{1}, \boldsymbol{y}_{1}, \boldsymbol{z}\right)\left\langle T^{(3)}\left(\boldsymbol{x}_{1}, \boldsymbol{z} ; \boldsymbol{z}, \boldsymbol{y}_{1} ; \boldsymbol{x}_{2}, \boldsymbol{y}_{2}\right)\right\rangle_{Y}\right) \\
& \left.+\kappa \alpha_{s}^{2} \frac{\boldsymbol{r}_{1}^{2} \boldsymbol{r}_{2}^{2}}{\left(\boldsymbol{r}_{1}+\boldsymbol{r}_{2}\right)^{2}}\left\langle T\left(\boldsymbol{x}_{1}, \boldsymbol{y}_{2}\right)\right\rangle_{Y} \delta^{(2)}\left(\boldsymbol{x}_{2}-\boldsymbol{y}_{1}\right)\right\} \\
+ & \{1 \leftrightarrow 2\} .
\end{aligned}
$$

Among the various terms in the r.h.s. of Eq. (6.7), those in the first two lines were already present in the corresponding Balitsky equation, while the term linear in $\langle T\rangle_{Y}$ in the third line (that we have written in the mixed notations of Eq. (2.2)) is a new term, which takes into account the effect of fluctuations. The unknown fudge factor $\kappa \sim \mathcal{O}(1)$ in this term has been introduced to parametrize our uncertainty concerning the precise relation between $T$ and $n$, cf. Eqs. (6.4) and (6.5). Note that this uncertainty does not affect the other, more standard, terms in the r.h.s. of Eq. (6.7), which come out the same as in the Balitsky-JIMWLK equations.

Let us discuss the properties of this new term in some detail. First, this is of order $\alpha_{s}^{2}\langle T\rangle$, so it is parametrically suppressed when $T \gg \alpha_{s}^{2}$ (since in that case already the uncorrelated piece $\langle T\rangle\langle T\rangle$ of $\left\langle T^{(2)}\right\rangle$ is much larger than the fluctuation term). In particular, the new term is certainly unimportant in the saturation regime, where Eq. (6.7) reduces to the corresponding Balitsky equation. On the other hand, the fluctuation term dominates in the very dilute regime where $\langle T\rangle \ll \alpha_{s}^{2}$, and thus is responsible for the growth of $\left\langle T^{(2)}\right\rangle$ in that regime. For instance, if we start with a target made of a single dipole at $Y=0$, then $\left\langle T^{(2)}\right\rangle_{0}=0$, and the rise of $\left\langle T^{(2)}\right\rangle$ in the early stages of the evolution is driven by the fluctuation term. A similar discussion applies to a rare high- $k_{\perp}$ (or small size) fluctuation generated in the wavefunction of an arbitrary target. We conclude that the usual BFKL evolution is not applicable in the dilute regime, in contrast to what one could naively expect. This failure will be further analyzed in Sect. 7 .

Consider also the geometry of the fluctuation term in the transverse plane, as manifest on Eq. (6.7). As anticipated in Sect. 2, for the external dipoles to feel the effect of fluctuations, they must be contiguous with each other, i.e., $\boldsymbol{x}_{2}=\boldsymbol{y}_{1}$ or $\boldsymbol{y}_{2}=\boldsymbol{x}_{1}$. Moreover, for contiguous dipoles of unequal transverse sizes, the importance of the fluctuation is controlled by the size of the smallest dipole: $\boldsymbol{r}_{1}^{2} \boldsymbol{r}_{2}^{2} /\left(\boldsymbol{r}_{1}+\boldsymbol{r}_{2}\right)^{2} \sim r_{<}^{2}$. This is so because of the geometry of dipole splitting (see Fig. 1) together with the fact that, in order to scatter, two dipoles have to overlap with each other. When a (target) dipole splits into 


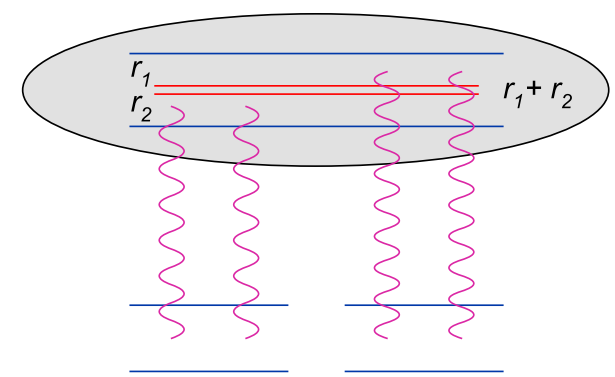

Fig. 8. Diagrammatic illustration of the fluctuation term in Eq. (6.7) : the original dipole with size $\boldsymbol{r}_{1}+\boldsymbol{r}_{2}$ splits at the time of the interaction into two new dipoles with sizes $\boldsymbol{r}_{1}$ and, respectively, $\boldsymbol{r}_{2}$, which then scatter off two external dipoles.

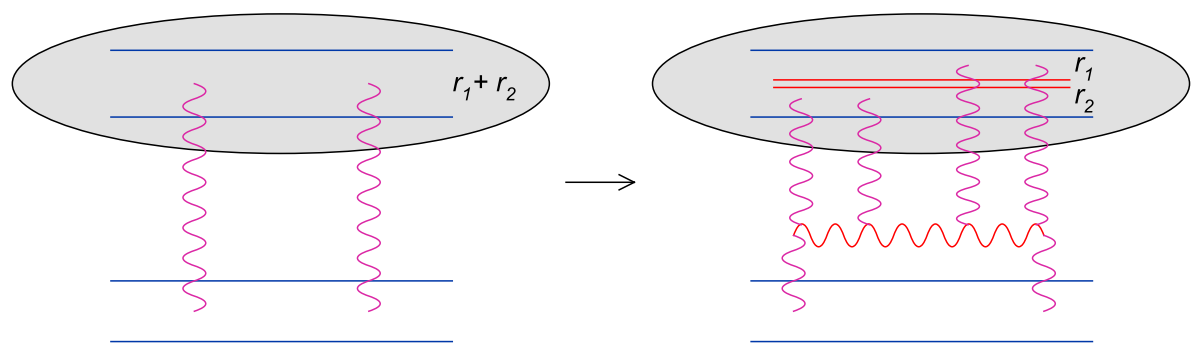

Fig. 9. Two steps in the evolution of the average scattering amplitude of a single dipole: the original amplitude (left) and its evolution after two steps (right).

two dipoles of very different sizes, the small child dipole is necessarily located near the edge of the parent dipole. Thus, for the scattering to take place, the impact parameter of the incoming small dipole should be located within a distance $r_{<}$from the edge of the parent dipole (of size $\left|\boldsymbol{r}_{1}+\boldsymbol{r}_{2}\right| \sim r_{>}$). This condition introduces the geometrical penalty factor $r_{<}^{2} / r_{>}^{2}$ manifest in the fluctuation term in Eq. (6.7). To conclude, the effects of the fluctuations are larger for incoming dipoles of comparable sizes.

Consider also the diagrammatic interpretation of the fluctuation term. This is illustrated in Fig. 8, where we represent the target as a collection of dipoles (as appropriate in the dilute regime), one out of which splits into two dipoles which then interact both with the external ones. This diagram is the dipole picture analog of the 2-to-4 splitting diagram in Fig. 4.g. Of course, the dipole picture cannot be used to also describe the non-linear effects in the previous equations, which correspond to saturation effects in the target wavefunction. To interpret the latter, we should rather resort to the CGC picture. In Fig. 9, we depict a particular two-step evolution of the single dipole amplitude $\langle T\rangle$, as emerging from Eqs. (3.3) and (6.7). The upper part of this diagram is the same as the splitting process in Fig. 8. The lower part describes the recombination of the four gluons resulting from that splitting into two; this is a diagram of the CGC formalism (compare to Fig. 2.f) which is encoded in the non-linear term in Eq. (3.3). The overall diagram in Fig. 9 represents the simplest pomeron loop generated by the present equations. 
It is straightforward to write down the generalization of Eq. (6.7) valid for an amplitude $\left\langle T^{(k)}\right\rangle$ with $k \geq 3$. This will be the corresponding (dipolar) Balitsky equation supplemented by fluctuation terms linear in $\left\langle T^{(k-1)}\right\rangle$ which account for the possibility that a pair of dipoles $\left(\boldsymbol{r}_{i}, \boldsymbol{r}_{j}\right)$ within $\left\langle T^{(k)}\right\rangle$ which are contiguous with each other be generated through the splitting of a unique dipole of size $\boldsymbol{r}_{i}+\boldsymbol{r}_{j}$ from $\left\langle T^{(k-1)}\right\rangle$. The ensuing hierarchy of equations, which generalizes the Balitsky hierarchy at large $N_{c}$ by including the effects of gluon number fluctuations in the dilute regime, represents our main result in this paper. As already manifest on Eq. (6.7), the equations in this hierarchy are afflicted with an uncertainty concerning the normalization of the fluctuation terms, which reflects the approximation (6.4) used in their derivation. As we shall explain in Sect. 7, this uncertainty does not affect the leading order predictions of these equations at very large $Y$ and small $\alpha_{s}$, but it may influence the subleading effects.

\subsection{The Langevin equation}

The equations for scattering amplitudes constructed in the previous subsection are similar to, although more complicated than, the general equations (4.9) for particle number correlations in the statistical toy model. In Sect. 4, we have seen that the hierarchy in Eq. (4.9) can be equivalently represented by a Langevin equation with an appropriate noise term. In what follows we shall show that, mediating some approximations which allow one to get rid of the impact parameter dependence of the amplitudes, a similar Langevin equation can be written also in QCD.

The Langevin equation will be seen to emerge naturally once that we succeed to rewrite the equations for the scattering amplitudes in a form quasi-local in $\boldsymbol{b}$. To that aim, we need to assume that the target is quasi-homogeneous over distances of the order of the size of the external dipoles. This is a reasonable approximation so long as we are interested in the local evolution towards saturation/unitarity, i.e., in the local 'blackening' of the target. In particular, this is sufficient for a study of the energy dependence of the saturation momentum. On the other hand, this approximation cannot be used to study the transverse expansion of the black disk, or to compute total cross-sections.

Under this assumption, all the terms in the previous equations except for the fluctuation terms become local in $\boldsymbol{b}$. For instance, the r.h.s. of Eq. (3.3)

for $\langle T(\boldsymbol{x}, \boldsymbol{y})\rangle \equiv\langle T(\boldsymbol{r}, \boldsymbol{b})\rangle$ involves $T(\boldsymbol{x}, \boldsymbol{z}) \equiv T\left(\boldsymbol{r}^{\prime}, \boldsymbol{b}+\frac{\boldsymbol{r}-\boldsymbol{r}^{\prime}}{2}\right)$ (with $\left.\boldsymbol{r}^{\prime}=\boldsymbol{x}-\boldsymbol{z}\right)$, which is approximated as $T\left(\boldsymbol{r}^{\prime}, \boldsymbol{b}\right)$. Then, Eq. (3.3) becomes: 


$$
\begin{aligned}
& \frac{\partial\langle T(\boldsymbol{r}, \boldsymbol{b})\rangle_{Y}}{\partial Y}=\frac{\bar{\alpha}_{s}}{2 \pi} \int d^{2} \boldsymbol{r}^{\prime} \frac{\boldsymbol{r}^{2}}{\boldsymbol{r}^{\prime 2}\left(\boldsymbol{r}-\boldsymbol{r}^{\prime}\right)^{2}} \\
& \left\langle-T(\boldsymbol{r}, \boldsymbol{b})+T\left(\boldsymbol{r}^{\prime}, \boldsymbol{b}\right)+T\left(\boldsymbol{r}-\boldsymbol{r}^{\prime}, \boldsymbol{b}\right)-T\left(\boldsymbol{r}^{\prime}, \boldsymbol{b}\right) T\left(\boldsymbol{r}-\boldsymbol{r}^{\prime}, \boldsymbol{b}\right)\right\rangle_{Y}
\end{aligned}
$$

which is indeed local in $\boldsymbol{b}$. Similarly, all the standard terms in the equation for $\left\langle T^{(2)}\left(\boldsymbol{r}_{1}, \boldsymbol{b}_{1} ; \boldsymbol{r}_{2}, \boldsymbol{b}_{2}\right)\right\rangle$ - that is, the terms in the first two lines in the r.h.s. of Eq. (6.7) - become bilocal in $\boldsymbol{b}_{1}$ and $\boldsymbol{b}_{2}$. But the fluctuation term there remains non-local, because of the $\delta$-functions imposing contiguity. We have indeed (compare to Eq. (5.16))

$$
\begin{aligned}
\frac{\left.\partial\left\langle T^{(2)}\left(\boldsymbol{r}_{1}, \boldsymbol{b}_{1} ; \boldsymbol{r}_{2}, \boldsymbol{b}_{2}\right)\right\rangle_{Y}\right|_{\text {fluct. }}=}{\partial Y} & \kappa \alpha_{s}^{2} \frac{\bar{\alpha}_{s}}{2 \pi} \frac{\boldsymbol{r}_{1}^{2} \boldsymbol{r}_{2}^{2}}{\left(\boldsymbol{r}_{1}+\boldsymbol{r}_{2}\right)^{2}}\left\langle T\left(\boldsymbol{r}_{1}+\boldsymbol{r}_{2}, \boldsymbol{b}_{1}\right)\right\rangle_{Y} \\
& \times \delta^{(2)}\left(\boldsymbol{b}_{1}-\boldsymbol{b}_{2}-\frac{\boldsymbol{r}_{1}+\boldsymbol{r}_{2}}{2}\right)+\{1 \leftrightarrow 2\},
\end{aligned}
$$

where we have approximated $T\left(\boldsymbol{r}_{1}+\boldsymbol{r}_{2}, \boldsymbol{b}_{1}-\frac{\boldsymbol{r}_{2}}{2}\right) \approx T\left(\boldsymbol{r}_{1}+\boldsymbol{r}_{2}, \boldsymbol{b}_{1}\right)$.

At this level it becomes natural to perform a Fourier transform to momentum space, by introducing

$$
\varphi(\boldsymbol{k}, \boldsymbol{b}) \equiv \int \frac{d^{2} \boldsymbol{r}}{2 \pi \boldsymbol{r}^{2}} \mathrm{e}^{-i \boldsymbol{k} \cdot \boldsymbol{r}} T(\boldsymbol{r}, \boldsymbol{b})
$$

Up to an overall normalization of $\mathcal{O}\left(1 / \bar{\alpha}_{s}\right), \varphi(\boldsymbol{k}, \boldsymbol{b})$ can be interpreted as the gluon phase-space occupation number, or the 'unintegrated gluon distribution' $[26,44]$. The benefit of using the momentum space is twofold: (i) After Fourier transform, the non-linear terms in the evolution equations (e.g., the term quadratic in $T$ in Eq. (6.8)) become local in $\boldsymbol{k}$. (ii) Since the fluctuation term (6.9) depends upon the transverse sizes $\boldsymbol{r}_{1}$ and $\boldsymbol{r}_{2}$ only through their sum $\boldsymbol{r}_{1}+\boldsymbol{r}_{2}$, its Fourier transform will be diagonal in momentum.

Specifically, Eq. (6.8) yields in momentum space :

$$
\frac{\partial\langle\varphi(\boldsymbol{k}, \boldsymbol{b})\rangle_{Y}}{\partial Y}=\bar{\alpha}_{s} \int \frac{d^{2} \boldsymbol{p}}{\pi} \frac{\boldsymbol{k}^{2}}{\boldsymbol{p}^{2}(\boldsymbol{k}-\boldsymbol{p})^{2}}\left\langle\frac{\boldsymbol{p}^{2}}{\boldsymbol{k}^{2}} \varphi(\boldsymbol{p}, \boldsymbol{b})-\frac{1}{2} \varphi(\boldsymbol{k}, \boldsymbol{b})\right\rangle_{Y}-\bar{\alpha}_{s}\left\langle\varphi^{2}(\boldsymbol{k}, \boldsymbol{b})\right\rangle_{Y}
$$

where the linear part is recognized as the BFKL equation for the unintegrated gluon distribution, while the non-linear term provides saturation.

Furthermore, the Fourier transform of the fluctuating contribution to $\left\langle T^{(2)}\right\rangle$, Eq. (6.9), is obtained as 


$$
\begin{aligned}
\left.\frac{\partial\left\langle\varphi\left(\boldsymbol{k}_{1}, \boldsymbol{b}_{1}\right) \varphi\left(\boldsymbol{k}_{2}, \boldsymbol{b}_{2}\right)\right\rangle_{Y}}{\partial Y}\right|_{\text {fluct. }}= & \bar{\alpha}_{s} 2 \kappa \alpha_{s}^{2} \delta^{(2)}\left(\boldsymbol{k}_{1}-\boldsymbol{k}_{2}\right) \\
& \times \int \frac{d^{2} \boldsymbol{r}}{2 \pi \boldsymbol{r}^{2}} \mathrm{e}^{-i \boldsymbol{k}_{1} \cdot \boldsymbol{r}}\left\langle T\left(\boldsymbol{r}, \boldsymbol{b}_{1}\right)\right\rangle \delta^{(2)}\left(\boldsymbol{b}_{1}-\boldsymbol{b}_{2}-\frac{\boldsymbol{r}}{2}\right),
\end{aligned}
$$

where the factor of 2 appears because the two terms in the r.h.s. of Eq. (6.9) have given identical contributions. Note that the exponential in the last integral selects $r \lesssim 1 / k_{1}$, thus the fluctuation term is important only for transverse separations $\left|\boldsymbol{b}_{1}-\boldsymbol{b}_{2}\right| \lesssim 1 / k_{1}$ between the two dipoles.

To also rewrite the fluctuation terms in a form which is local in $\boldsymbol{b}$, we shall proceed to a coarse-graining. This is physically motivated, since an external dipole of size $r$ cannot distinguish details in the target on a transverse scale much smaller than $r$. That is, the gluon (or dipole) distribution measured by the external dipole is necessarily averaged in $\boldsymbol{b}$ over a disk of radius $r$ around its impact parameter. After Fourier transform to momentum space, this corresponds, by the uncertainty principle, to a coarse-graining over an area $\sim 1 / k^{2}$. To implement this, we shall divide the impact parameter space into cells of area $1 / k^{2}$, and average the scattering amplitudes over any such a cell. Consider a particular cell with center at $\boldsymbol{b}_{i}$. Averaged quantities within that cell are then defined as:

$$
\varphi_{i}(\boldsymbol{k}) \equiv k^{2} \int_{\Sigma_{i}(k)} d^{2} \boldsymbol{b} \varphi(\boldsymbol{k}, \boldsymbol{b}), \quad \varphi_{i}^{2}(\boldsymbol{k}) \equiv k^{2} \int_{\Sigma_{i}(k)} d^{2} \boldsymbol{b} \varphi^{2}(\boldsymbol{k}, \boldsymbol{b})
$$

etc., where $\Sigma_{i}(k) \sim 1 / k^{2}$ is the area of the cell. In fact, given our previous assumption that the target is quasi-homogeneous over the area covered by the dipole, it is clear that the averaging above is tantamount to simply replacing $\varphi(\boldsymbol{k}, \boldsymbol{b})$ everywhere within a cell by its value at the center of that cell: $\varphi_{i}(\boldsymbol{k}) \approx$ $\varphi\left(\boldsymbol{k}, \boldsymbol{b}_{i}\right)$. Therefore, $\varphi_{i}^{2}(\boldsymbol{k}) \approx\left[\varphi_{i}(\boldsymbol{k})\right]^{2}$, so, by itself, this coarse-graining does not introduce additional correlations.

The coarse-graining is therefore trivial for the standard (BFKL + nonlinear) terms in the evolution equations: it amounts to replacing $\varphi(\boldsymbol{k}, \boldsymbol{b}) \rightarrow$ $\varphi_{i}(\boldsymbol{k})$ (for a dipole hitting the target in cell $i$ ) in any of those terms. The non-locality of the BFKL kernel poses no special difficulties for this coarsegraining since, e.g., the integral over $\boldsymbol{p}$ in Eq. (6.11) is dominated by momenta $p \sim k$. Then Eq. (6.11) implies that the equation for the average amplitude $\left\langle\varphi_{i}(\boldsymbol{k})\right\rangle$ in cell $i$ involves the double-scattering amplitude within the same cell, $\left\langle\varphi_{i}^{2}(\boldsymbol{k})\right\rangle$. To compute this, it is therefore enough to study the scattering of two external dipoles which hit the target within the same cell. This is also the interesting case for a study of fluctuations: as discussed after Eq. (6.12), the fluctuations vanish when the external dipoles fall in different cells.

Consider therefore the equation satisfied by $\left\langle\varphi_{i}\left(\boldsymbol{k}_{1}\right) \varphi_{i}\left(\boldsymbol{k}_{2}\right)\right\rangle$. The coarsegraining is non-trivial only for the fluctuation term, in which case it implies 
(note that $\boldsymbol{k}_{1}=\boldsymbol{k}_{2}$ in this term, cf. Eq. (6.12)):

$$
\begin{gathered}
k_{1}^{4} \int_{\Sigma_{i}\left(k_{1}\right)} d^{2} \boldsymbol{b}_{1} \int_{\Sigma_{i}\left(k_{1}\right)} d^{2} \boldsymbol{b}_{2} \int \frac{d^{2} \boldsymbol{r}}{2 \pi \boldsymbol{r}^{2}} \mathrm{e}^{-i \boldsymbol{k}_{1} \cdot \boldsymbol{r}}\left\langle T\left(\boldsymbol{r}, \boldsymbol{b}_{1}\right)\right\rangle \delta^{(2)}\left(\boldsymbol{b}_{1}-\boldsymbol{b}_{2}-\frac{\boldsymbol{r}}{2}\right) \\
=k_{1}^{4} \int_{\Sigma_{i}\left(k_{1}\right)} d^{2} \boldsymbol{b}_{1} \int \frac{d^{2} \boldsymbol{r}}{2 \pi \boldsymbol{r}^{2}} \mathrm{e}^{-i \boldsymbol{k}_{1} \cdot \boldsymbol{r}}\left\langle T\left(\boldsymbol{r}, \boldsymbol{b}_{1}\right)\right\rangle=k_{1}^{2}\left\langle\varphi_{i}(\boldsymbol{k})\right\rangle .
\end{gathered}
$$

The integration over $\boldsymbol{b}_{2}$ has been used to remove the $\delta$-function (whose support is indeed included in $\Sigma_{i}\left(k_{1}\right)$ ), while the integration over $\boldsymbol{b}_{1}$ has served together with Eq. (6.10) to construct $\left\langle\varphi_{i}(\boldsymbol{k})\right\rangle$.

Let us summarize here the first two equations of the hierarchy, as applying to the scattering within cell $i$ :

$$
\frac{\partial\left\langle\varphi_{i}(\boldsymbol{k})\right\rangle_{Y}}{\partial Y}=\bar{\alpha}_{s} \int \frac{d^{2} \boldsymbol{p}}{2 \pi} \frac{\boldsymbol{k}^{2}}{\boldsymbol{p}^{2}(\boldsymbol{k}-\boldsymbol{p})^{2}}\left\langle 2 \frac{\boldsymbol{p}^{2}}{\boldsymbol{k}^{2}} \varphi_{i}(\boldsymbol{p})-\varphi_{i}(\boldsymbol{k})\right\rangle_{Y}-\bar{\alpha}_{s}\left\langle\varphi_{i}^{2}(\boldsymbol{k})\right\rangle_{Y},
$$

and, respectively,

$$
\begin{aligned}
\frac{\partial\left\langle\varphi_{i}\left(\boldsymbol{k}_{1}\right) \varphi_{i}\left(\boldsymbol{k}_{2}\right)\right\rangle_{Y}}{\partial Y}=\bar{\alpha}_{s}\{ & \int \frac{d^{2} \boldsymbol{p}}{2 \pi} \frac{\boldsymbol{k}_{1}^{2}}{\boldsymbol{p}^{2}\left(\boldsymbol{k}_{1}-\boldsymbol{p}\right)^{2}}\left\langle\left(2 \frac{\boldsymbol{p}^{2}}{\boldsymbol{k}_{1}^{2}} \varphi_{i}(\boldsymbol{p})-\varphi_{i}\left(\boldsymbol{k}_{1}\right)\right) \varphi_{i}\left(\boldsymbol{k}_{2}\right)\right\rangle_{Y} \\
& -\left\langle\varphi_{i}^{2}\left(\boldsymbol{k}_{1}\right) \varphi_{i}\left(\boldsymbol{k}_{2}\right)\right\rangle_{Y}, \\
& \left.+\kappa \alpha_{s}^{2} \delta^{(2)}\left(\boldsymbol{k}_{1}-\boldsymbol{k}_{2}\right) \boldsymbol{k}_{1}^{2}\left\langle\varphi_{i}\left(\boldsymbol{k}_{1}\right)\right\rangle_{Y}\right\} \\
& +\{1 \leftrightarrow 2\} .
\end{aligned}
$$

Clearly, these equations are (quasi)local in $\boldsymbol{b}$ in the sense that they involve only operators pertaining to cell $i$. Needless to say, this property holds also for the higher equations in the hierarchy. Within the present, quasi-homogeneous, approximation, different cells in the impact parameter space evolve independently from each other.

It is now straightforward to identify the Langevin equation which generates the same correlations as the hierarchy at cell $i$. This reads (from now on, we omit the cell index $i$, as there is no coupling between different cells):

$$
\begin{gathered}
\frac{1}{\bar{\alpha}_{s}} \frac{\partial \varphi(\boldsymbol{k})}{\partial Y}=\int \frac{d^{2} \boldsymbol{p}}{2 \pi} \frac{\boldsymbol{k}^{2}}{\boldsymbol{p}^{2}(\boldsymbol{k}-\boldsymbol{p})^{2}}\left(2 \frac{\boldsymbol{p}^{2}}{\boldsymbol{k}^{2}} \varphi(\boldsymbol{p})-\varphi(\boldsymbol{k})\right)-\varphi^{2}(\boldsymbol{k}) \\
+\sqrt{2 \kappa \alpha_{s}^{2} \varphi(\boldsymbol{k})} \nu(\boldsymbol{k})
\end{gathered}
$$

where $\nu(\boldsymbol{k}, Y)$ is a Gaussian white noise $:\langle\nu(\boldsymbol{k}, Y)\rangle=0$ and 


$$
\left\langle\nu\left(\boldsymbol{k}_{1}, Y_{1}\right) \nu\left(\boldsymbol{k}_{2}, Y_{2}\right)\right\rangle=\frac{1}{\bar{\alpha}_{s}} \delta\left(Y_{1}-Y_{2}\right) \delta^{(2)}\left(\boldsymbol{k}_{1}-\boldsymbol{k}_{2}\right) \boldsymbol{k}_{1}^{2} .
$$

Eq. (6.17) must be understood with a rapidity discretization prescription of the Ito type, as explicitly shown in Eq. (4.11).

Eq. (6.17) should be compared to Eq. (3.1) which, we recall, is the generating equation of the Balitsky hierarchy. Clearly, Eq. (3.1) is the same as the (Fourier transform of the) deterministic part of Eq. (6.17), but the latter involves also the noise term responsible for fluctuations. In that respect, Eq. (6.17) is closer to the Langevin equation (4.10) for the statistical toy model. Note, however, that in contrast to Eq. (4.10), the noise term in the corresponding equation (6.17) for QCD does not vanish at saturation. This reflects the fact that, in our previous analysis of QCD, we did not include the effect of fluctuations on the recombination terms (an effect which would go beyond the dipole picture that we have used to describe fluctuations in QCD). However, this omission is truly harmless: In the high density regime at/near saturation, fluctuations are anyway unimportant, and there is no need to suppress them explicitly ${ }^{13}$. In fact, it is rather obvious on Eq. (6.17) that the effects of the noise term are important only so long as $\varphi(\boldsymbol{k}) \lesssim \alpha_{s}^{2}$.

\section{Physical discussion}

The Langevin equation (6.17) turns out to be the natural generalization to QCD of the stochastic Fisher-Kolmogorov-Petrovsky-Piscounov (sFKPP) equation (see the Appendix), to which it reduces in a standard approximation to the BFKL kernel known as the 'diffusion approximation'. This will be discussed in Sect. 7.1. Then, in Sects. 7.2 and 7.3, we shall use some known results about the sFKPP equation $[38,45,46]$ (see also Refs. $[39,40]$ for recent reviews and more references) in order to explore the physical consequences of Eq. (6.17) for QCD. In particular, in Sect. 7.2, we shall make the connection with the physical discussion and the results in Ref. [34]. Then, in Sect. 7.3, we shall discover a rather dramatic consequence of the fluctuations, namely the breakdown of the BFKL approximation in the regime where the average gluon density is small.

\footnotetext{
$\overline{13}$ Numerical simulations within the context of statistical physics $[38,45]$ have confirmed that the sFKPP equation, in which the noise term has as a coefficient $\sqrt{\alpha \varphi(1-\varphi)}$ - with $\alpha \ll 1$ and $\varphi=1$ at saturation - and the so-called Reggeon model, where the coefficient of the noise is simply $\sqrt{\alpha \varphi}$, lead indeed to identical results for the measured correlations.
} 


\subsection{Relation with the sFKPP equation}

In order to understand the relation between our Langevin equation Eq. (6.17) in QCD and the sFKPP equation in statistical physics, it is useful to first recall a few facts about the deterministic part of Eq. (6.17), as obtained after neglecting the noise term there. If we also neglect correlations in the initial conditions, then this deterministic part is simply the momentum-space version of the BK equation, which can be more compactly rewritten as:

$$
\partial_{Y} \varphi(\rho, Y)=\bar{\alpha}_{s} \chi\left(-\partial_{\rho}\right) \varphi-\bar{\alpha}_{s} \varphi^{2}
$$

where $\rho \equiv \ln k^{2} / k_{0}^{2}$ (with $k_{0}$ some arbitrary momentum scale of reference), $\chi(\gamma)=2 \psi(1)-\psi(\gamma)-\psi(1-\gamma)$ is the Mellin transform of the BFKL kernel, and $\chi\left(-\partial_{\rho}\right)$ is an integro-differential operator defined via the series expansion of $\chi(\gamma)$ (see below). In a series of papers [29], Munier and Peschanski have argued that Eq. (7.1) is in the same universality class as the Fisher-KolmogorovPetrovsky-Piscounov (FKPP) equation, which appears as a mean field approximation to a variety of stochastic problems in chemistry, physics, and biology $[39,47]$. To understand this correspondence, notice that in the dilute regime at high transverse momenta, $k^{2} \gg Q_{s}^{2}(Y)$ or $\rho \gg \rho_{s}(Y)$, with $\rho_{s}(Y) \equiv \ln Q_{s}^{2}(Y) / k_{0}^{2}$, we have $\phi \ll 1$, so the dominant behavior of $\varphi(\rho, Y)$ is determined by the linear part of Eq. (7.1), which is the BFKL equation [1]. One thus finds that the dominant dependencies upon $\rho$ and $Y$ can be isolated out into an exponential factor:

$$
\varphi(\rho, Y)=e^{-\gamma_{0}\left(\rho-\lambda_{0} \bar{\alpha}_{s} Y\right)} \psi(\rho, Y)
$$

where $\gamma_{0}$ and $\lambda_{0}$ are pure numbers determined by the BFKL kernel as [2] :

$$
\gamma_{0} \chi^{\prime}\left(\gamma_{0}\right)=\chi\left(\gamma_{0}\right), \quad \lambda_{0}=\frac{\chi\left(\gamma_{0}\right)}{\gamma_{0}}
$$

which implies $\gamma_{0} \approx 0.63$ and $\lambda_{0}=\chi^{\prime}\left(\gamma_{0}\right) \approx 4.88$, and the function $\psi(\rho, Y)$ is comparatively slowly varying, so its behavior can be studied by using a limited expansion of the operator $\chi\left(-\partial_{\rho}\right)$ around $\chi\left(\gamma_{0}\right)$ :

$$
\begin{aligned}
\chi\left(-\partial_{\rho}\right) & =\chi\left(\gamma_{0}\right)+\chi^{\prime}\left(\gamma_{0}\right)\left(-\partial_{\rho}-\gamma_{0}\right)+\frac{1}{2} \chi^{\prime \prime}\left(\gamma_{0}\right)\left(-\partial_{\rho}-\gamma_{0}\right)^{2} \ldots \\
& \approx-\lambda_{0} \partial_{\rho}+D_{0}\left(-\partial_{\rho}-\gamma_{0}\right)^{2}
\end{aligned}
$$

where $D_{0} \equiv \chi^{\prime \prime}\left(\gamma_{0}\right) / 2$ and in writing the second line we have also used Eq. (7.3). The approximation which consists in keeping only the terms to 
second order in this expansion, as explicitly shown in the second line above, is generally referred to as the "diffusion approximation", and is equivalent to a saddle point approximation to the solution to BFKL equation in Mellin space. $\mathrm{v}$ In the case of BFKL equation, one can use Eqs. (7.2) and (7.4) to show that $\psi(\rho, Y)$ obeys a diffusion equation. For the non-linear BK equation (7.1), one can argue that the main effect of the non-linear term is to introduce an absorptive boundary condition on the diffusion equation for $\psi$ [27]. Alternatively, one can study the full non-linear equation which emerges within the diffusion approximation:

$$
\partial_{Y} \varphi(\rho, Y)=\bar{\alpha}_{s}\left(-\lambda_{0} \partial_{\rho}+D_{0}\left(-\partial_{\rho}-\gamma_{0}\right)^{2}\right) \varphi-\bar{\alpha}_{s} \varphi^{2}
$$

Munier and Peschanski have observed that, up to a linear change of variables and an appropriate rescaling of $\varphi$, Eq. (7.5) is the same as the FKPP equation:

$$
\partial_{t} u(x, t)=\partial_{x}^{2} u(x, t)+u(x, t)(1-u(x, t)),
$$

which at large times describes an uniformly translating front which propagates from the stable state $u=1$ into the unstable one $u=0$ and decays exponentially at large $x$ (far ahead the front) $[39,47]$. In QCD, this front is already visible on Eq. (7.2), which at large $Y$ describes a traveling wave located at $\rho=\rho_{s}(Y) \approx \lambda_{0} \bar{\alpha}_{s} Y$ which has an exponential slope $\gamma_{0}$ and propagates in 'time' $\bar{\alpha}_{s} Y$ with uniform velocity $\lambda_{0}$. (This behavior is the origin of geometric scaling [35] for the BK equation [26,27].) By using known properties of the FKPP equation [39] or, alternatively, by solving the diffusion equation for $\psi$ with an absorptive boundary condition [27], one can deduce the dominant corrections to the velocity and the shape of the front due to non-linearities. For large $Y$ and $\rho \gg \rho_{s}(Y)$, one finds $\psi(\rho, Y) \sim\left(\rho-\rho_{s}(Y)\right) / Y^{3 / 2}$, and therefore:

$$
\frac{d \rho_{s}(Y)}{d Y} \simeq \bar{\alpha} \lambda_{0}-\frac{3}{2 \gamma_{0}} \frac{1}{Y}
$$

Returning to the original Langevin equation (6.17) and assuming that the dominant $\rho$-behavior of the solution is still given by the exponential $e^{-\gamma_{0} \rho}$ (which turns out to be right indeed), one can use again the diffusion approximation (7.4) to deduce a simplified form of the equation $\left(\tau \equiv \bar{\alpha}_{s} Y\right)$ :

$$
\partial_{\tau} \varphi(\rho, \tau)=-\lambda_{0} \partial_{\rho} \varphi+D_{0}\left(-\partial_{\rho}-\gamma_{0}\right)^{2} \varphi-\varphi^{2}+\sqrt{2 \kappa \alpha_{s}^{2} \varphi} \nu(\rho, \tau),
$$

with the Gaussian white noise :

$$
\langle\nu(\rho, \tau)\rangle=0, \quad\left\langle\nu(\rho, \tau) \nu\left(\rho^{\prime}, \tau^{\prime}\right)\right\rangle=\frac{1}{\pi} \delta\left(\tau-\tau^{\prime}\right) \delta\left(\rho-\rho^{\prime}\right)
$$


Up to a simple change of variables, the equation above is essentially the same as the sFKPP equation [40]:

$$
\partial_{t} u(x, t)=\partial_{x}^{2} u+u(1-u)+\sqrt{\frac{2}{N} u(1-u)} \nu(x, t) .
$$

In this analogy, the density of particles per site $u(x, t)$ in the stochastic particle model corresponds to the scattering amplitude (or unintegrated gluon distribution) $\varphi(\rho, \tau)$ in QCD, and $1 / \alpha_{s}^{2}$ plays the same role as $N$ (the number of particles per site at saturation), which is reasonable since we recall that $n \sim 1 / \alpha_{s}^{2}$ is also the dipole occupation number at saturation. Therefore, the mean field approximation emerges in the limit where the occupation numbers are large, i.e., $N \rightarrow \infty$ for the particle model and $\alpha_{s}^{2} \rightarrow 0$ in QCD.

Note finally the limitations of this correspondence, as inherent in the use of the diffusion approximation. First, Eq. (7.8) predicts that behind the front $\varphi$ saturates at a constant value $\varphi=\gamma_{0}^{2} D_{0}$ (or $u=1$ for Eq. (7.10)), whereas the original Langevin rather yields $\varphi(k, Y) \approx \ln \left(Q_{s}(Y) / k\right)$ [i.e., $\varphi(\rho, Y) \approx$ $\left.\left(\rho_{s}(Y)-\rho\right) / 2\right]$ for $\rho \ll \rho_{s}(Y)$, as most easily seen by using Eq. (6.10) together with the fact that $T(r)=1$ for $r \gg 1 / Q_{s}(Y)$. Second, for fixed $Y$ and sufficiently large $\rho$ (essentially, such that $\rho-\rho_{s}(Y) \gtrsim \rho_{s}(Y)[26]$ ), the BFKL 'anomalous dimension' $1-\gamma$ approaches to zero, which signals the transition to a regime dominated by the DGLAP dynamics. Clearly, in this regime it is not possible to expand the BFKL kernel around the saturation exponent $\gamma_{0}$, as we did in Eq. (7.4). Thus, we do not expect the simplified equation (7.8) to describe correctly the transition to the DGLAP regime.

\subsection{Some results from sFKPP equation and their consequences for QCD}

Even if somehow simpler than the original Lagevin equation (6.17), the sFKPP equation (7.8) (or (7.10)) remains complicated, because of the simultaneous presence of the non-linear term for recombination and of the multiplicative noise term. Fortunately, this equation has been extensively studied in relation with problems in statistical physics - mostly through numerical simulations, but also via some analytical methods - , with results that we shall briefly describe here and then adapt to the QCD problem at hand. In applying these results to QCD, one should however keep in mind the possible limitations of the correspondence between Eqs. (6.17) and (7.10), as mentioned at the end of the previous subsection.

It has been rigorously demonstrated [46] that the front generated by the sFKPP equation (7.10) is compact : For any $t$, there exists a $x_{r}(t)$ such that

$u(x, t)=0$ for $x>x_{r}(t)$, and also a $x_{l}(t)$ such that $u(x, t)=1$ for $x<$ 
$x_{l}(t)$. One expects a similar property for Eq. (6.17), although in that case the transition to the saturation region behind the front may not be as sharp, because the coefficient of the corresponding noise term does not vanish at saturation.

Numerical simulations show that for large but finite $N$, the front generated by Eq. (7.10) propagates with an asymptotic velocity $v_{N}$ which is smaller than the corresponding velocity $v_{0}=2$ for the FKPP equation (7.6). Moreover, with increasing $N$ the convergence of $v_{N}$ towards $v_{0}$ is extremely slow: $v_{0}-v_{N} \sim$ $1 / \ln ^{2} N$ when $N \gg 1$. For the corresponding QCD problem, this implies the following asymptotic $Y$-dependence of the saturation scale :

$$
\lambda_{s} \equiv \lim _{\tau \rightarrow \infty} \frac{d \rho_{s}(\tau)}{d \tau} \simeq \lambda_{0}-\frac{\mathcal{C}}{\ln ^{2}\left(1 / \alpha_{s}^{2}\right)} \quad \text { when } \quad \alpha_{s} \ll 1,
$$

(recall that $\tau=\bar{\alpha}_{s} Y$ ). Thus, with decreasing $\alpha_{s}$, the saturation exponent $\lambda_{s}$ converges only slowly towards the respective mean-field value in Eq. (7.3).

Since the noise term in Eq. (6.17) is important only for relatively small $\varphi \lesssim \alpha_{s}^{2}$, we expect the shape of an individual front (in its comoving frame) to be rather well described by the solution (7.2) to the deterministic equation (7.1) at all the points where $\varphi \gg \alpha_{s}^{2}$ (or $u \gg 1 / N$ for Eq. (7.10)). This is seen indeed in the numerical simulations. One can then estimate the width of the front as follows: It is the noise term in Eq. (6.17) which abruptly cuts down the growth of $\varphi$ ahead of the front, but this requires $\varphi$ to be as small as $\alpha_{s}^{2}$. Since in the front region behind the tip $\varphi$ behaves like $\varphi \simeq e^{-\gamma_{0}\left(\rho-\rho_{s}\right)}$, cf. Eq. (7.2), we conclude that $\varphi$ decreases from 1 to $\alpha_{s}^{2}$ over a range $\rho-\rho_{s} \sim\left(1 / \gamma_{0}\right) \ln \left(1 / \alpha_{s}^{2}\right)$, which should be a good estimate for the width of the front.

However, because of the fluctuations inherent in the noise term, different realizations of the same evolution will lead to an ensemble of fronts which all have the same shape, but are displaced with respect to each other along the $\rho$-axis. That is, the position $\rho_{s}$ of the front is itself a random variable, characterized by an expectation value $\left\langle\rho_{s}(\tau)\right\rangle$, which for large $\tau$ increases according to Eq. (7.11) (since this is the common asymptotic behavior of all the fronts in the ensemble), and also by a dispersion $\sigma^{2} \equiv\left\langle\rho_{s}^{2}\right\rangle-\left\langle\rho_{s}\right\rangle^{2}$, which is expected to rise linearly with $\tau: \sigma^{2}(\tau) \sim D_{\text {fr }} \tau$ (since the front executes a random walk around its average position). The numerical simulations to the sFKPP equation confirm this behavior, and show that the front diffusion coefficient $D_{\text {fr }}$ scales like $1 / \ln ^{3} N$ when $N \gg 1$. For QCD, this in turn implies:

$$
D_{\mathrm{fr}} \simeq \frac{\mathcal{D}}{\ln ^{3}\left(1 / \alpha_{s}^{2}\right)} \quad \text { when } \quad \alpha_{s} \ll 1,
$$

which vanishes, as expected, when $\alpha_{s} \rightarrow 0$, but only very slowly. 
Note that the results (7.11) and (7.12) are only logarithmically sensitive to the coefficient of $\mathcal{O}\left(\alpha_{s}^{2}\right)$ of the noise term in Eq. (6.17), or (7.8). Thus, the leading order estimates in the limit $\alpha_{s} \rightarrow 0$, as shown in Eqs. (7.11) and (7.12), are not affected by our uncertainty concerning the fudge factor $\kappa$ in the noise. On the other hand, the next-to-leading order correction already will be sensitive to the precise coefficient under the log, and thus to $\kappa$ (since, e.g., $\left.1 / \ln ^{2}\left(a / \kappa \alpha_{s}^{2}\right) \approx 1 / \ln ^{2}\left(1 / \alpha_{s}^{2}\right)-2 \ln (a / \kappa) / \ln ^{3}\left(1 / \alpha_{s}^{2}\right)\right)$.

The results of the sFKPP equation alluded to above turn out to be consistent with numerical and analytic studies of the stochastic particle models whose mean field approximation is the FKPP equation (7.6). This agreement should not come as a surprise: As shown in the Appendix, the sFKPP equation emerges precisely as the continuum description of such discrete particle models. In particular, in that context, Brunet and Derrida [48] have given a simple heuristic argument which explains the $1 / \ln ^{2} N$ scaling of the velocity correction $v_{0}-v_{N}$ at large $N$, and also allows one to compute the corresponding coefficient $\mathcal{C}$. Namely, they have observed that, in the presence of discreteness, diffusion should replace local growth as the main mechanism for front propagation: Indeed, in order for the growth term (the linear term in $u(x, t)$ in Eq. (7.6)) to be effective, there must be at least one particle per bin (or lattice site). Thus, the only way that a particle can move to an originally empty bin ahead of the front is via diffusion from the previously occupied bins on its left. To mimic that, Brunet and Derrida proposed a modified deterministic equation obtained by inserting a cutoff $\theta(u-1 / N)$ in the growth term in Eq. (7.6) (since a density $u \sim 1 / N$ corresponds to a site occupation number of $\mathcal{O}(1))$. A simple analysis of this equation then implies [48] $v_{0}-v_{N} \simeq \mathcal{C} / \ln ^{2} N$ for $N \gg 1$, with a value for $\mathcal{C}$ which is indeed consistent with the numerical studies of both particle models and the sFKPP equation. After translation to the QCD problem of interest here, this in turn implies:

$$
\lambda_{s} \simeq \lambda_{0}-\frac{\pi^{2} \gamma_{0} \chi^{\prime \prime}\left(\gamma_{0}\right)}{2 \ln ^{2}\left(1 / \alpha_{s}^{2}\right)} \quad \text { when } \quad \alpha_{s} \ll 1 \text {. }
$$

It so happens that the QCD coefficient $\mathcal{C}=\pi^{2} \gamma_{0} \chi^{\prime \prime}\left(\gamma_{0}\right) / 2$ is numerically large, $\mathcal{C} \approx 150$, so the corrective term in the equation above can be trusted only for extremely small values of $\alpha_{s}$, which are physically unrealistic. To our knowledge, there is no analytic argument allowing one to understand the scaling (7.12) of the front diffusion coefficient, or to compute the coefficient $\mathcal{D}$ there. (See however the discussion in Sect. 4 of Ref. [40].)

Furthermore, in the context of QCD, the above results (7.11) and (7.12) of the sFKPP equation corroborate the conclusions obtained in Ref. [34] through an analogy between the high-energy problem in QCD and some specific particle models in statistical physics. This in turn demonstrates that the Langevin equation (6.17), or, more generally, the stochastic equations presented in Sect. 
6.2, provide the correct evolution law underlying the physical picture put forward in Ref. [34]. By (numerically) solving these equations, one can now go beyond the analysis in Ref. [34] and study the evolution for realistic (nonasymptotic) values of $Y$ and $\alpha_{s}^{2}$.

\subsection{Front diffusion and the breakdown of the BFKL approximation}

It has been argued in Ref. [34] that, as a consequence of the diffusive wandering of the front, the 'geometric scaling' property characteristic of the individual fronts - i.e., the fact that a particular front realization propagates as a travelling wave, $T(\rho, Y) \approx T\left(\rho-\rho_{s}(Y)\right)$ (cf. Eq. (7.2)), so that its shape in the comoving frame is not changed under the evolution - is actually broken after averaging over the statistical ensemble of fronts generated by the stochastic evolution up to large $Y$. In what follows, we shall demonstrate that the consequences of the front diffusion are in fact even more dramatic, as they entail the breakdown of the BFKL approximation in the dilute regime, where this approximation is usually assumed to work ${ }^{14}$. What we mean by that more precisely is that, at very high energies, the standard BFKL equation (i.e., the linearized part of Eqs. (3.3) or (7.1)) fails to correctly describe the evolution of the average scattering amplitude $\langle T(\rho)\rangle_{Y}$ even in the regime where this amplitude is small, $\langle T(\rho)\rangle_{Y} \ll 1$. This is so because, in the presence of fluctuations and for sufficiently large $Y$, average quantities like $\langle T\rangle_{Y}$ or $\left\langle T^{(2)}\right\rangle_{Y}$ are dominated by those fronts within the statistical ensemble which are at saturation for the values of $\rho$ of interest, and this even when $\rho$ is well above the average saturation momentum $\left\langle\rho_{s}\right\rangle_{Y}$, where $\langle T(\rho)\rangle_{Y} \ll 1$ indeed.

Note that in this subsection we return to the notation $T$ (rather than $\varphi)$ for the amplitude, that is, we prefer to work in coordinate space, where $\rho=\ln \left(1 / r^{2} k_{0}^{2}\right)$. Then, for a given front realization, with saturation momentum $\rho_{s}(Y)$, the scattering amplitude saturates to $T=1$ behind the front, and takes the scaling form $T(\rho, Y) \simeq e^{-\gamma_{0}\left(\rho-\rho_{s}\right)}$ within a finite range in $z$ ahead of the front. (Recall that the front is compact.) For the present purposes, it suffices to describe this behavior with the simple interpolation:

$$
T\left(\rho, \rho_{s}\right)= \begin{cases}1 & \text { for } \rho \leq \rho_{s} \\ \exp \left[-\gamma_{0}\left(\rho-\rho_{s}\right)\right] & \text { for } \rho \geq \rho_{s}\end{cases}
$$

which neglects the compact nature of the front; this is harmless since, as we shall see, the tail of the distribution at large $z$ does not contribute to average quantities in the regime of interest.

Because of the diffusive nature of the front wandering, the values of $\rho_{s}$ are

\footnotetext{
${ }^{14}$ We are grateful to $\mathrm{Al}$ Mueller for helping us clarifying this point.
} 
distributed according to the probability density

$$
P\left(\rho_{s}\right)=\frac{1}{\sqrt{\pi} \sigma} \exp \left[-\frac{\left(\rho_{s}-\left\langle\rho_{s}\right\rangle\right)^{2}}{\sigma^{2}}\right],
$$

where $\left\langle\rho_{s}\right\rangle \simeq \lambda_{s} \bar{\alpha}_{s} Y$ and $\sigma^{2} \simeq D_{\mathrm{fr}} \bar{\alpha}_{s} Y$, as discussed before. Then the average amplitude $\langle T\rangle$ is determined by

$$
\left\langle T\left(\rho,\left\langle\rho_{s}\right\rangle\right)\right\rangle=\int_{-\infty}^{\infty} d \rho_{s} P\left(\rho_{s}\right) T\left(\rho, \rho_{s}\right),
$$

and higher-point correlations can be computed similarly. For simplicity, in what follows we shall restrict ourselves to higher correlations evaluated at equal points, e.g., $\left\langle T^{(2)}(\rho, \rho)\right\rangle \equiv\left\langle T^{2}(\rho)\right\rangle$. Then, one can obtain $\left\langle T^{n}\right\rangle$ by simply replacing $\gamma_{0} \rightarrow n \gamma_{0}$ in the subsequent formulae for $\langle T\rangle$.

By using Eqs.(7.14) and Eq.(7.15) it is straightforward to show that

$$
\langle T\rangle=\frac{1}{2} \operatorname{Erfc}\left(\frac{z}{\sigma}\right)+\frac{1}{2} \exp \left(\frac{\gamma_{0}^{2} \sigma^{2}}{4}-\gamma_{0} z\right)\left[2-\operatorname{Erfc}\left(\frac{z}{\sigma}-\frac{\gamma_{0} \sigma}{2}\right)\right],
$$

where $z \equiv \rho-\left\langle\rho_{s}\right\rangle$ and $\operatorname{Erfc}(\mathrm{x})$ is the complimentary error function, for which we recall that

$$
\operatorname{Erfc}(x)= \begin{cases}2-\frac{\exp \left(-x^{2}\right)}{\sqrt{\pi} x} & \text { for } x \ll-1 \\ 1 & \text { for } x=0 \\ \frac{\exp \left(-x^{2}\right)}{\sqrt{\pi} x} & \text { for } x \gg 1 .\end{cases}
$$

For what follows it is important to notice that the two terms in the r.h.s. of Eq.(7.17) arise from the saturating piece and, respectively, the exponentially decaying piece, of Eq.(7.14).

The behavior of $\langle T\rangle$ as a function of $z$ depends upon the competition between $\sigma$ (the width of the Gaussian distribution of the fronts) and $1 / \gamma_{0}$, which characterizes the exponential decay of the individual fronts. Since $\sigma$ grows like $\sqrt{Y}$ whereas $\gamma_{0} \sim \mathcal{O}(1)$, one may conclude that the typical situation at high energy is such that $\sigma \gg 1 / \gamma_{0}$. But the diffusion coefficient (7.12) vanishes when $\alpha_{s} \rightarrow 0$, even though only slowly, so for sufficiently small $\alpha_{s}$ at fixed $Y$ one can imagine also the situation where $\sigma \ll 1 / \gamma_{0}$. So, we shall consider both cases here:

- $\underline{\underline{1} \ll 1 / \gamma_{0}}$

In this regime, the front diffusion should play no role, and this is indeed what we find. Specifically, when $z \ll-\sigma$, by making use of Eq.(7.18) one sees 
that the first term in Eq.(7.17) gives 1, while the second one is negligible. On the contrary, when $z \gg \sigma$, the first term is small while the second one gives $\exp \left(-\gamma_{0} z\right)$. Thus, not surprisingly, one finds that the average amplitude $\langle T\rangle$ retains the single event profile, except in the short interval $|z| \lesssim \sigma$ where it gets smoothed. The BFKL dynamics, characterized by the anomalous dimension $\gamma_{0}$, is still visible, and the mean field approximation holds since $\left\langle T^{n}\right\rangle \simeq\langle T\rangle^{n}$.

\section{- $\underline{\underline{ } \gg 1 / \gamma_{0}}$}

In this regime, the correlations are dominated by fluctuations, and the BFKL behavior is washed out everywhere except at extremely large distances ahead of the front (where however the present approximations cannot be trusted since, e.g., the compact support property of the front, and also higher correlations in the diffusion of the front, may play a role).

Specifically, for all values of $z$ such that $z \ll \gamma_{0} \sigma^{2}$ one finds that $\langle T\rangle$ is dominated by the first term in Eq.(7.17):

$$
\langle T\rangle \simeq \frac{1}{2} \operatorname{Erfc}\left(\frac{z}{\sigma}\right) \quad \text { for } \quad-\infty<z \ll \gamma_{0} \sigma^{2}
$$

which, as mentioned earlier, is the contribution from the saturating pieces of the single events, and thus is independent of $\gamma_{0}$. This estimate holds, in particular, in the range $\sigma \ll z \ll \gamma_{0} \sigma^{2}$ where $\langle T\rangle$ is small, $\langle T\rangle \ll 1$, yet very different from the corresponding BFKL prediction. In particular, there is no trace of geometric scaling, in agreement with Refs. [33,34].

In fact, within the whole range in $z$ in which Eq.(7.19) is valid, the higher correlations $\left\langle T^{n}\right\rangle$ are given by this same expression, that is

$$
\left\langle T^{n}\right\rangle \simeq\langle T\rangle \quad \text { for } \quad-\infty<z \ll \gamma_{0} \sigma^{2},
$$

which signals a total breakdown of the mean field approximation, except in the saturation regime where $\langle T\rangle \simeq 1$. One can even find a window within which $\left\langle T^{n}\right\rangle$ varies very slowly, namely:

$$
\left\langle T^{n}\right\rangle \simeq\langle T\rangle \simeq \frac{1}{2} \quad \text { for } \quad|z| \ll \sigma
$$

The different behaviors encountered when increasing $\sigma$ (and thus $Y$ ) are illustrated on the example of $\langle T\rangle$ and $\left\langle T^{2}\right\rangle$ in Fig. 10.

Let us conclude this section, and also the paper, with a final remark concerning the role of fluctuations in the high-energy evolution in QCD, and, in particular, on their interplay with saturation. It has been a recurrent theme in this paper that fluctuations in the dilute regime act as a seed for the growth of higher-point correlations which then, through their subsequent evolution, 

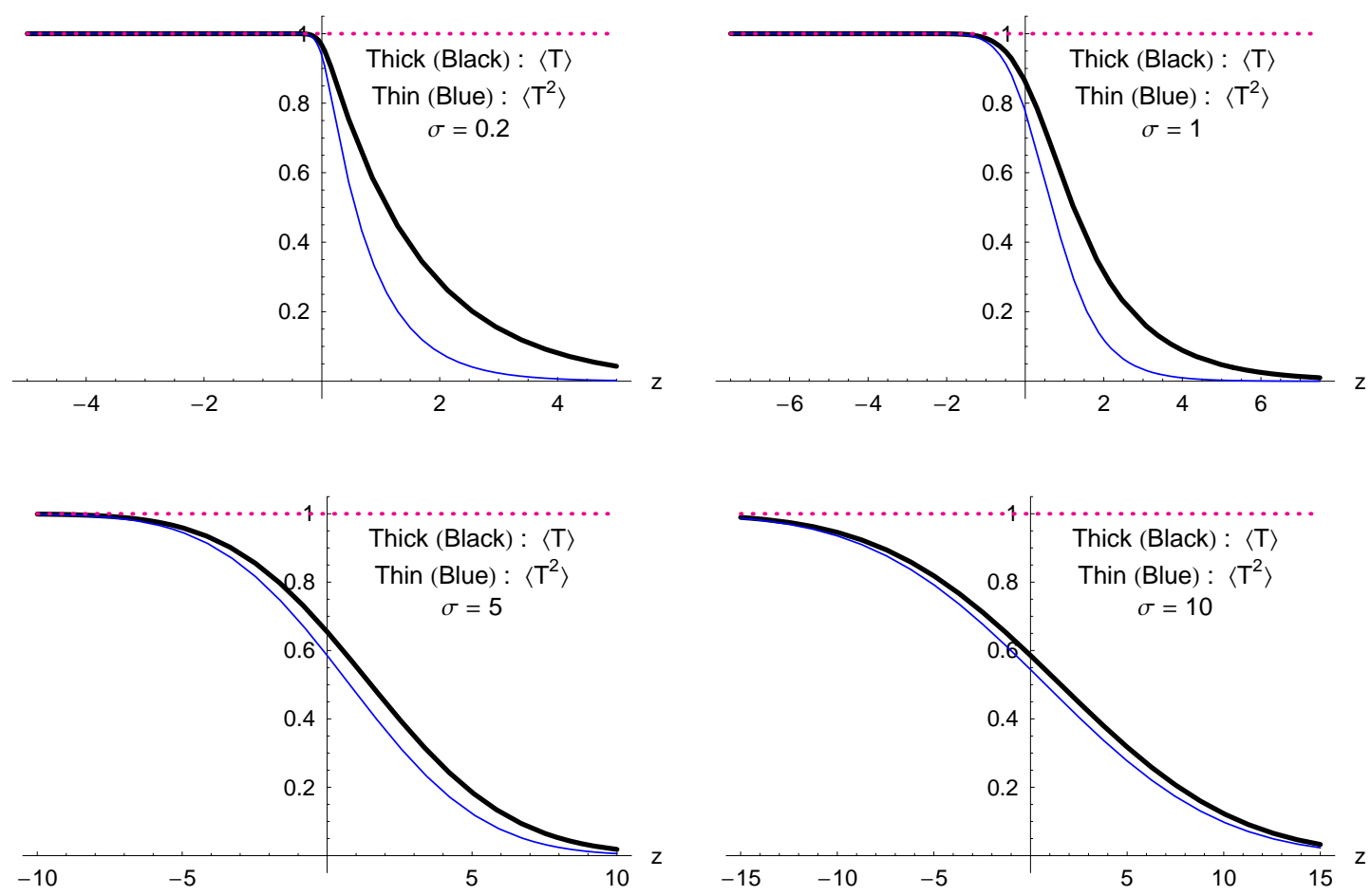

Fig. 10. Evolution of $\langle T\rangle$ and $\left\langle T^{2}\right\rangle$ with increasing $\sigma$.

generate the non-linear terms responsible for saturation. This is the (more or less) expected part of the scenario, in which fluctuations and saturation are important at different ends of the spectrum, and the amplitude $T(\rho, Y)$ is reasonably well described by the mean field approximation (the BK equation) everywhere except at the tip of the distribution where $T$ is very small: $T \lesssim \alpha_{s}^{2}$. This picture is indeed what emerges from the dynamical equation (6.17), but only so long as one considers a single front realization (i.e., a single event). If, on the other hand, one considers the statistical ensemble of fronts - which is what one needs to do in order to compute average quantities - , then the dispersion of the fronts due to fluctuations has a rather unexpected consequence, which is to render the correlation functions sensitive to saturation even in the (formally) weak scattering regime, where the average scattering amplitude is small, and thus wash out the normal BFKL behavior.

\section{Acknowledgments}

The ideas presented in this paper have crystallized over a rather lengthy period of time, during which we have benefited from illuminating discussions and useful suggestions from several colleagues. In particular, the efforts developed by one of us (E.I.) together with Kazu Itakura and Al Mueller towards understanding 'pomeron loops' in the context of non-linear evolution have certainly had an important influence on the gestation of the work presented here. 
We are particularly grateful to Larry McLerran and Al Mueller for helping us clarifying several important points in the present analysis. We are equally grateful to Jean-Paul Blaizot, François Gelis, Kazu Itakura, Robi Peschanski and Gavin Salam for their comments on this manuscript and other insightful remarks. We further acknowledge useful conversations with Ian Balitsky, Yoshitaka Hatta, Yuri Kovchegov, Misha Lublinsky, Stephane Munier, Arif Shoshi, Raju Venugopalan, Samuel Wallon, and Stephen Wong.

\section{A A one-dimensional reaction-diffusion model}

In this Appendix we shall study a much more sophisticated model, than the one we did in Sect. 4, which shares a lot of similar features with QCD. We consider a system whose state is described by the number of particles A at every site $i$ in a one-dimensional lattice; each state is of the form $\left\{n_{i}\right\}=$ $\left\{n_{1}, n_{2}, \ldots, n_{i}, \ldots\right\}$, where $n_{i}$ is the number of particles at site $i$. The dynamics contains three possible processes:

- A particle can split locally into two at a rate $\alpha$, i.e.

$$
\mathrm{A}_{i} \stackrel{\alpha}{\rightarrow} \mathrm{A}_{i}+\mathrm{A}_{i}
$$

- Two particles can recombine locally into one at a rate $2 \beta_{0}$, i.e.

$$
\mathrm{A}_{i}+\mathrm{A}_{i} \stackrel{2 \beta_{0}}{\longrightarrow} \mathrm{A}_{i}
$$

- A particle can diffuse to a neighboring site at a rate $\mu$, i.e.

$$
\mathrm{A}_{i} \stackrel{\mu}{\rightarrow} \mathrm{A}_{i+1}, \quad \mathrm{~A}_{i} \stackrel{\mu}{\rightarrow} \mathrm{A}_{i-1} .
$$

Our task is to derive the evolution equation for $\left\langle n_{i}\right\rangle$ and $\left\langle n_{i} n_{j}\right\rangle$ from the master equation, which we do not write explicitly here, but the corresponding changes in probabilities will be obvious from the analysis below. Then we will study the continuous limit, which will lead us to the necessity of requiring normal-ordering, and we will end this Appendix by introducing the, relevant to the problem, Langevin equation.

Since splitting and recombination take place locally, their contribution to the time evolution of $\left\langle n_{i}\right\rangle$ is easy to obtain. For both these processes, one can have either gain or loss under a step $d Y$, depending on the number of parti-

cles in the initial configuration. There are two terms arising from the splitting part $d P\left(\left\{n_{i}\right\} ; Y\right) /\left.d Y\right|_{\alpha}$ (all the other splittings which occur at lattice points different than the one we measure, i.e. the $i$-th one, cancel each other): 
$\circ$ Loss $\quad-\alpha n_{i} P\left(\ldots, n_{i}, \ldots ; Y\right)$

○ Gain $\quad+\alpha\left(n_{i}-1\right) P\left(\ldots, n_{i}-1, \ldots ; Y\right)$,

and two terms from the recombination part $d P\left(\left\{n_{i}\right\} ; Y\right) /\left.d Y\right|_{\beta_{0}}$

$\circ \operatorname{Loss} \quad-\beta_{0} n_{i}\left(n_{i}-1\right) P\left(\ldots, n_{i}, \ldots ; Y\right)$

○ Gain $\quad+\beta_{0}\left(n_{i}+1\right) n_{i} P\left(\ldots, n_{i}+1, \ldots ; Y\right)$

To calculate $d\left\langle n_{i}\right\rangle /\left.d Y\right|_{\alpha, \beta_{0}}$, we appropriately shift the $n$ 's wherever needed to obtain

$$
\left.\frac{d\left\langle n_{i}\right\rangle}{d Y}\right|_{\alpha, \beta_{0}}=\sum_{\left\{n_{i}\right\}} P\left(\left\{n_{i}\right\} ; Y\right) F_{\alpha, \beta_{0}}\left(\left\{n_{i}\right\}\right),
$$

where, after very simple algebra, one can find that $F_{\alpha, \beta_{0}}\left(\left\{n_{i}\right\}\right)=\alpha n_{i}-\beta_{0}\left(n_{i}^{2}-\right.$ $\left.n_{i}\right)$. Therefore we arrive at

$$
\left.\frac{d\left\langle n_{i}\right\rangle}{d Y}\right|_{\alpha, \beta_{0}}=\alpha\left\langle n_{i}\right\rangle-\beta_{0}\left[\left\langle n_{i}^{2}\right\rangle-\left\langle n_{i}\right\rangle\right] .
$$

Now let us also study in detail the evolution of $\left\langle n_{i}\right\rangle$ due to diffusion. Under a step $d Y$ in time, the diffusion can drive an particle from site $i$ to $i \pm 1$. Again, in all cases we can have either gain or loss depending on the number of particles in the initial configuration. Overall there are eight terms arising from the diffusive part $d P\left(\left\{n_{i}\right\} ; Y\right) /\left.d Y\right|_{\mu}$ :

○ Loss

$$
\begin{array}{lrl}
-\mu n_{i} P\left(\ldots, n_{i-1}, n_{i}, n_{i+1}, \ldots ; Y\right) & \text { from } & i \rightarrow i-1 \\
-\mu n_{i} P\left(\ldots, n_{i-1}, n_{i}, n_{i+1}, \ldots ; Y\right) & \text { from } & i \rightarrow i+1 \\
-\mu n_{i-1} P\left(\ldots, n_{i-1}, n_{i}, n_{i+1}, \ldots ; Y\right) & \text { from } & i-1 \rightarrow i \\
-\mu n_{i+1} P\left(\ldots, n_{i-1}, n_{i}, n_{i+1}, \ldots ; Y\right) & \text { from } & i+1 \rightarrow i
\end{array}
$$

o Gain

$$
\begin{array}{lll}
+\mu\left(n_{i}+1\right) P\left(\ldots, n_{i-1}-1, n_{i}+1, n_{i+1}, \ldots ; Y\right) & \text { from } & i \rightarrow i-1 \\
+\mu\left(n_{i}+1\right) P\left(\ldots, n_{i-1}, n_{i}+1, n_{i+1}-1, \ldots ; Y\right) & \text { from } & i \rightarrow i+1 \\
+\mu\left(n_{i-1}+1\right) P\left(\ldots, n_{i-1}+1, n_{i}-1, n_{i+1}, \ldots ; Y\right) & \text { from } & i-1 \rightarrow i \\
+\mu\left(n_{i+1}+1\right) P\left(\ldots, n_{i-1}, n_{i}-1, n_{i+1}+1, \ldots ; Y\right) & \text { from } & i+1 \rightarrow i
\end{array}
$$

As before, in order to calculate $d\left\langle n_{i}\right\rangle /\left.d Y\right|_{\mu}$, we appropriately shift the $n$ 's 
wherever needed to obtain

$$
\left.\frac{d\left\langle n_{i}\right\rangle}{d Y}\right|_{\mu}=\sum_{\left\{n_{i}\right\}} P\left(\left\{n_{i}\right\} ; Y\right) F_{\mu}\left(\left\{n_{i}\right\}\right),
$$

with $F_{\mu}\left(\left\{n_{i}\right\}\right)=\mu\left(n_{i+1}+n_{i-1}-2 n_{i}\right)$. Therefore we arrive at

$$
\left.\frac{d\left\langle n_{i}\right\rangle}{d Y}\right|_{\mu}=\mu\left\langle\nabla^{2} n_{i}\right\rangle
$$

where, for reasons that will become obvious shortly, we introduced the shorthand notation

$$
\nabla^{2} n_{i} \equiv n_{i+1}+n_{i-1}-2 n_{i+1} .
$$

Now, when considering the pair density we need to study the three separate cases $\left\langle n_{i}^{2}\right\rangle,\left\langle n_{i} n_{i+1}\right\rangle$ and $\left\langle n_{i} n_{j}\right\rangle$ for $|i-j| \geq 2$. Here we shall skip the derivation, which can be done by following the same steps of the $\left\langle n_{i}\right\rangle$ case, and only give the final set of the evolution equations which read

$$
\begin{aligned}
\left.\frac{d\left\langle n_{i} n_{j}\right\rangle}{d Y}\right|_{\alpha}= & \alpha\left\langle 2 n_{i} n_{j}+\delta_{i j} n_{i}\right\rangle \\
\left.\frac{d\left\langle n_{i} n_{j}\right\rangle}{d Y}\right|_{\beta_{0}}= & -\beta_{0}\left\langle n_{i}^{2} n_{j}+n_{i} n_{j}^{2}-2 n_{i} n_{j}-\delta_{i j}\left(n_{i}^{2}-n_{i}\right)\right\rangle, \\
\left.\frac{d\left\langle n_{i} n_{j}\right\rangle}{d Y}\right|_{\mu}= & \mu\left\langle n_{i} \nabla^{2} n_{j}+n_{i} \nabla^{2} n_{j}\right. \\
& \left.+\delta_{i j}\left(\nabla^{2} n_{i}+4 n_{i}\right)-\left(\delta_{i, j-1}+\delta_{i, j+1}\right)\left(n_{i}+n_{j}\right)\right\rangle .
\end{aligned}
$$

Let's turn our attention to the continuous limit. Let the lattice spacing be equal to $\Delta$, the coordinate $x=i \Delta$ and the particle single and pair densities $\bar{n}(x)=n_{i} / \Delta$ and $\bar{n}\left(x_{1}\right) \bar{n}\left(x_{2}\right)=n_{i} n_{j} / \Delta^{2}$. In order to be economical in the notation we shall drop the bars immediately. Then the single density equation becomes

$$
\frac{\partial\langle n(x)\rangle}{\partial Y}=\alpha\langle n(x)\rangle-\beta_{0} \Delta\left[\left\langle n^{2}(x)\right\rangle-\frac{1}{\Delta}\langle n(x)\rangle\right]+\mu \Delta^{2} \frac{\partial^{2}\langle n(x)\rangle}{\partial x^{2}}+\mathcal{O}\left(\mu \Delta^{4}\right) .
$$

Notice that the next to last term justifies the shorthand notation adopted in Eq.(A.8). Considering first this diffusion term, we naturally impose that $\mu \Delta^{2} \equiv D=$ fixed in the $\Delta \rightarrow 0$ limit. Then all the higher order terms can be ignored. Similarly, we require $\beta_{0} \Delta \equiv \beta=$ fixed for the recombination term. It is only the recombination process which generates potential singularities in the continuous limit, since it involves the pair density. However, even though the second term in the square bracket becomes divergent, we expect the first term to do so in the same limit in such a way to cancel the singularity. Indeed, the 
whole bracket corresponds to the $x_{1} \rightarrow x_{2}$ limit of the average normal-ordered pair density

$$
n^{(2)}\left(x_{1}, x_{2}\right) \equiv\left\langle: n\left(x_{1}\right) n\left(x_{2}\right):\right\rangle=\left\langle n\left(x_{1}\right) n\left(x_{2}\right)\right\rangle-\delta\left(x_{1}-x_{2}\right)\left\langle n\left(x_{1}\right)\right\rangle
$$

and thus we finally arrive at

$$
\frac{\partial n^{(1)}(x)}{\partial Y}=\alpha n^{(1)}(x)-\beta n^{(2)}(x, x)+D \frac{\partial^{2} n^{(1)}(x)}{\partial x^{2}}
$$

with the obvious identification $n^{(1)}(x) \equiv\langle n(x)\rangle$. Of course, in the absence of recombination, these subtleties would have not appeared. But they do appear for all the processes, i.e. even for splitting and diffusion, when we try to take the continuous limit in the equation of the pair density. As an illustration, let's concentrate on the diffusion contribution. When we consider the $|i-j| \geq 2$ case we arrive at an equation with no singularities, since all the terms in the second line of Eq.(A.11) vanish. But those linear terms do not vanish when we start from the discrete equation with $|i-j| \leq 1$ (while the quadratic terms do not change). In fact, they are divergent in the limit $\Delta \rightarrow 0$. Moreover they are "scheme-dependent", in the sense that, even though all cases $|i-j| \leq 1$ lead to an equation for $\left\langle n^{2}(x)\right\rangle$, this equation is not uniquely obtained; it depends on the particular discrete equation that one starts with. We will not write all the details here, but we shall give the most divergent terms. We easily find that

$$
\left.\frac{\partial\left\langle n^{2}(x, x)\right\rangle}{\partial Y}\right|_{\operatorname{div}}= \begin{cases}4 \frac{D}{\Delta^{3}}\langle n(x)\rangle+\mathcal{O}\left(\frac{1}{\Delta^{2}}\right) & \text { from } i=j \\ -2 \frac{D}{\Delta^{3}}\langle n(x)\rangle+\mathcal{O}\left(\frac{1}{\Delta^{2}}\right) & \text { from } i=j \pm 1\end{cases}
$$

From the previous discussion, we expect that the divergencies will disappear when we consider the evolution of normal ordered densities. Indeed, using Eq.(A.13) and

$$
\begin{aligned}
n^{(3)}\left(x_{1}, x_{2}, x_{3}\right)= & \left\langle n\left(x_{1}\right) n\left(x_{2}\right) n\left(x_{3}\right)\right\rangle-\left[\delta\left(x_{1}-x_{2}\right)\left\langle n\left(x_{1}\right) n\left(x_{3}\right)\right\rangle+\text { perm. }\right] \\
& +2 \delta\left(x_{1}-x_{2}\right) \delta\left(x_{1}-x_{3}\right)\left\langle n\left(x_{1}\right)\right\rangle
\end{aligned}
$$

and along with Eq.(A.14) we obtain a unique and finite evolution equation of the pair density in the elegant form

$$
\begin{aligned}
\frac{\partial n^{(2)}\left(x_{1}, x_{2}\right)}{\partial Y}= & \left\{\alpha\left[n^{(2)}\left(x_{1}, x_{2}\right)+\delta\left(x_{1}-x_{2}\right) n^{(1)}\left(x_{1}\right)\right]\right. \\
& -\beta\left[n^{(3)}\left(x_{1}, x_{1}, x_{2}\right)+\delta\left(x_{1}-x_{2}\right) n^{(2)}\left(x_{1}, x_{1}\right)\right] \\
& \left.+D n^{(1)}\left(x_{2}\right) \frac{\partial^{2} n^{(1)}\left(x_{1}\right)}{\partial x_{1}^{2}}\right\}+\{1 \leftrightarrow 2\}
\end{aligned}
$$


In principle one could follow the same procedure to derive the evolution equation for the general $\kappa$-point function $n^{(\kappa)}\left(x_{1}, \ldots, x_{\kappa}\right)$ or understand the form by looking at the first two equations, (A.14) and (A.17), which we just derived.

We shall not write the full hierarchy here, but the easiest and most rewarding way to derive it is from the corresponding Langevin equation. This is simply the generalization of the Langevin equation we saw in Sec. 4 and reads

$$
\frac{\partial \tilde{n}(x)}{\partial Y}=\alpha \tilde{n}(x)-\beta \tilde{n}^{2}(x)+D \frac{\partial^{2} \tilde{n}(x)}{\partial x^{2}}+\sqrt{2\left[\alpha \tilde{n}(x)-\beta \tilde{n}^{2}(x)\right]} \nu(x, Y),
$$

where the noise satisfies $\langle\nu(x, Y)\rangle=0$ and $\left\langle\nu\left(x_{1}, Y\right) \nu\left(x_{2}, Y^{\prime}\right)\right\rangle=\delta\left(x_{1}-\right.$ $\left.x_{2}\right) \delta\left(Y-Y^{\prime}\right)$. Notice that $\tilde{n}(x)$ saturates at the value $\alpha / \beta$. The hierarchy generated by Eq.(A.18) is equivalent to the one of the model we studied, provided we simply identify $\left\langle\tilde{n}\left(x_{1}\right) \ldots \tilde{n}\left(x_{\kappa}\right)\right\rangle$ with $n^{(\kappa)}\left(x_{1}, \ldots, x_{\kappa}\right)$. One sees that in a straightforward way by deriving Eqs.(A.14) and (A.17) from Eq.(A.18), while a formal proof can be found in [38].

For our convenience, and in order to make a closer analogy to the QCD problem (where $T$ saturates to one), we can make the change of variables $\tilde{n} \rightarrow(\alpha / \beta) u, Y \rightarrow t / \alpha, D \rightarrow \alpha D$ and $\nu \rightarrow \sqrt{\alpha} \nu$. Then the Langevin equation becomes

$$
\frac{\partial u(x)}{\partial t}=u(x)-u^{2}(x)+D \frac{\partial^{2} u(x)}{\partial x^{2}}+\sqrt{\frac{2 \beta}{\alpha}\left[u(x)-u^{2}(x)\right]} \nu(x, t),
$$

with $\langle\nu(x, t)\rangle=0$ and $\left\langle\nu\left(x_{1}, t\right) \nu\left(x_{2}, t^{\prime}\right)\right\rangle=\delta\left(x_{1}-x_{2}\right) \delta\left(t-t^{\prime}\right)$. Now $u(x)$ saturates at the value 1 .

\section{References}

[1] L.N. Lipatov, Sov. J. Nucl. Phys. 23 (1976) 338; E.A. Kuraev, L.N. Lipatov and V.S. Fadin, Zh. Eksp. Teor. Fiz 72, 3 (1977) (Sov. Phys. JETP 45 (1977) 199); Ya.Ya. Balitsky and L.N. Lipatov, Sov. J. Nucl. Phys. 28 (1978) 822.

[2] L.V. Gribov, E.M. Levin, and M.G. Ryskin, Phys. Rept. 100 (1983) 1.

[3] A.H. Mueller and J. Qiu, Nucl. Phys. B268 (1986) 427.

[4] J.-P. Blaizot and A. H. Mueller, Nucl. Phys. B289 (1987) 847.

[5] A. H. Mueller, Nucl. Phys. B415 (1994) 373; A. H. Mueller and B. Patel, Nucl. Phys. B425 (1994) 471.

[6] A. H. Mueller, Nucl. Phys. B437 (1995) 107.

[7] I. Balitsky, Nucl. Phys. B463 (1996) 99; Phys. Rev. Lett. 81 (1998) 2024; Phys. Lett. B518 (2001) 235; High-energy QCD and Wilson lines, hep-ph/0101042. 
[8] E. Levin and M. Lublinsky, "Balitsky's hierarchy from Mueller's dipole model and more about target correlations", arXiv:hep-ph/0411121.

[9] L. McLerran and R. Venugopalan, Phys. Rev. D49 (1994) 2233; ibid. 49 (1994) 3352; ibid. 50 (1994) 2225.

[10] Yu.V. Kovchegov, Phys. Rev. D54 (1996), 5463; Phys. Rev. D55 (1997), 5445.

[11] J. Jalilian-Marian, A. Kovner, L. McLerran, H. Weigert, Phys. Rev. D55 (1997) 5414 .

[12] J. Jalilian-Marian, A. Kovner, A. Leonidov and H. Weigert, Nucl. Phys. B504 (1997) 415; Phys. Rev. D59 (1999) 014014.

[13] E. Iancu, A. Leonidov and L. McLerran, Nucl. Phys. A692 (2001) 583; Phys. Lett. B510 (2001) 133; E. Ferreiro, E. Iancu, A. Leonidov and L. McLerran, Nucl. Phys. A703 (2002) 489.

[14] E. Iancu and L. McLerran, Phys. Lett. B510 (2001) 145.

[15] E. Iancu, A. Leonidov and L. McLerran, The Colour Glass Condensate: An Introduction, hep-ph/0202270 Published in QCD Perspectives on Hot and Dense Matter, Eds. J.-P. Blaizot and E. Iancu, NATO Science Series, Kluwer, 2002.

E. Iancu and R. Venugopalan, The Color Glass Condensate and High Energy Scattering in QCD, hep-ph/0303204. Published in Quark-Gluon Plasma 3, Eds. R. C. Hwa and X.-N. Wang, World Scientific, 2003.

[16] H. Weigert, Nucl. Phys. A703 (2002) 823.

[17] Yu. V. Kovchegov, Phys. Rev. D60 (1999), 034008; ibid. D61 (2000) 074018.

[18] K. Rummukainen and H. Weigert, Nucl. Phys. A739 (2004) 183.

[19] E. Levin and K. Tuchin, Nucl. Phys. B573 (2000) 833; Nucl. Phys. $\mathbf{6 9 9 1}$ (2001) 779; Nucl. Phys. A693 (2001) 787.

[20] N. Armesto and M. Braun, Eur. Phys. J. C20 (2001) 517; ibid. C22 (2001) 351.

[21] K. Golec-Biernat, L. Motyka, and A.M. Staśto, Phys. Rev. D65 (2002) 074037.

[22] E. Levin and M. Lublinsky, Phys. Lett. B521 (2001) 233; Eur. Phys. J. C22 (2002) 647; M. Lublinsky, Eur. Phys. J. C21 (2001) 513.

[23] K. Golec-Biernat and A.M. Stasto, Nucl. Phys. B668 (2003) 345.

[24] J. L. Albacete, N. Armesto, A. Kovner, C. A. Salgado and U. A. Wiedemann, Phys. Rev. Lett. 92 (2004) 082001.

[25] J. L. Albacete, N. Armesto, J. G. Milhano, C. A. Salgado and U. A. Wiedemann, "Numerical analysis of the Balitsky-Kovchegov equation with running coupling: dependence of the saturation scale on nuclear size and rapidity", arXiv:hep-ph/0408216

[26] E. Iancu, K. Itakura, and L. McLerran, Nucl. Phys. A708 (2002) 327. 
[27] A. H. Mueller and D.N. Triantafyllopoulos, Nucl. Phys. B640 (2002) 331.

[28] D.N. Triantafyllopoulos, Nucl. Phys. B648 (2003) 293.

[29] S. Munier and R. Peschanski, Phys. Rev. Lett. 91 (2003) 232001 ; Phys. Rev. D69 (2004) 034008; ibid. D70 (2004) 077503.

[30] G.P. Salam, Nucl. Phys. B449 (1995) 589; Nucl. Phys. B461 (1996) 512.

[31] A. H. Mueller and G.P. Salam, Nucl. Phys. B475 (1996) 293.

[32] E. Iancu and A.H. Mueller, Nucl. Phys. A730 (2004) 494.

[33] A.H. Mueller and A.I. Shoshi, Nucl. Phys. B692 (2004) 175.

[34] E. Iancu, A.H. Mueller, and S. Munier, "Universal behavior of QCD amplitudes at high energy from general tools of statistical physics", arXiv:hep-ph/0410018

[35] A. M. Stasto, K. Golec-Biernat and J. Kwiecinski, Phys. Rev. Lett. 86 (2001) 596.

[36] E. Iancu and A.H. Mueller, Nucl. Phys. A730 (2004) 460.

[37] E. Levin and M. Lublinsky, Nucl. Phys. A730 (2004) 191.

[38] L. Pechenik and H. Levine, Phys. Rev. E69 (1999) 3893.

[39] For a recent review, see W. Van Saarloos, Phys. Rep. 386 (2003) 29 arXiv:cond-mat/0308540.

[40] For a recent review, see D. Panja, Phys. Rep. 393 (2004) 87 arXiv:cond-mat/0307363.

[41] R. A. Janik, R. Peschanski, Phys. Rev. D70 (2004) 094005.

[42] R. A. Janik, "QCD saturation in the dipole sector with correlations", arXiv:hep-ph/0409256.

[43] D. Panja, W. Van Saarloos, Phys. Rev. E $66 \quad$ (2002) 036206 arXiv:cond-mat/0109528.

[44] E. Iancu, K. Itakura, and D.N. Triantafyllopoulos, Nucl. Phys. A742 (2004) 182.

[45] E. Moro, Phys. Rev. E70 (2004) 045102(R).

[46] C. Mueller, R.B. Sowers, . J. Funct. Anal. 128 (1995) 439.

[47] R. A. Fisher, Ann. Eugenics 7 (1937) 355; A. Kolmogorov, I. Petrovsky, and N. Piscounov, Moscou Univ. Bull. Math. A1 (1937) 1.

[48] E. Brunet and B. Derrida, Phys. Rev. E56 (1997) 2597; Comp. Phys. Comm. 121-122 (1999) 376; J. Stat. Phys. 103 (2001) 269. 